\title{
Confidence-based Robust Optimisation Using Multi-objective Meta-heuristics
}

\author{
Seyedali Mirjalili, Andrew Lewis, Jin Song Dong \\ Institute for Integrated and Intelligent Systems, Griffith University, Brisbane, 4111 QLD, Australia \\ *seyedali.mirjalili@griffithuni.edu.au
}

\begin{abstract}
Robust optimisation refers to the process of finding optimal solutions that have the lowest sensitivity to possible perturbations. In a multi-objective search space the robust optimal solutions should have the least dispersion on all of the objectives, making it a more challenging problem than in a single-objective search space. This paper establishes a novel and cheap technique for finding robust optimal solutions called confidence-based robust multi-objective optimisation. This approach uses a novel, modified Pareto dominance operator to differentiate search agents of meta-heuristics based on both level of robustness and confidence. The proposed confidence-based Pareto dominance allows us to design different confidence-based robust optimisation variants of meta-heuristics based on different methods. As a case study, robust Multi-Objective Particle Swarm Optimisation is equipped with the proposed operator to produce Confidence-based Robust Multi-Objective Particle Swarm Optimisation. A set of specific test functions and performance indicators is employed for benchmarking the Confidence-based Robust Multi-Objective Particle Swarm Optimisation. The results show that the proposed method is able to confidently and reliably find robust optimal solutions without significant extra computational burden. The paper also considers finding robust Pareto optimal front for a marine propeller design problem to demonstrate the applicability of the approach proposed in solving computationally expensive real-world problems with unknown true robust Pareto optimal fronts.
\end{abstract}

Keywords: Robust optimisation, Confidence-based robust optimisation, Handling uncertainty

\section{Introduction}

One of the most important concepts in the optimisation of real engineering problems is robustness. Robust optimisation refers to the process of combining good performance with low sensitivity to possible perturbations. Uncertainties are unavoidable in the real world and can be classified in three categories: those affecting parameters, operating conditions, and outputs. One of the most common type of uncertainties is perturbation of parameters, in which the design parameters are varied after finding the optimal design(s).

Another important property of real engineering problems is multi-objectiveness. Optimisation in a search space with multiple objectives is more difficult than a search space with single objective. In the former type of problems, a set of best solutions representing the trade-offs between the objectives must be found. In the latter type of problems, however, one solution should be found as the only best solution.

Similarly, robust optimisation in a single-objective search space is also different from that in a search space with multiple objectives. In a single-objective search space, there is one robust solution with the least variations. In a multi-objective search space, however, robust optimal solutions belong to a set of optimal solutions called the robust Pareto optimal set representing the robust trade-offs between the multiple objectives.

Multi-objective meta-heuristics were employed to perform robust optimisation in 2006 [1]. Deb and Gupta established two different approaches for robust optimisation in multi-objective search spaces: expectation-based and variance-based methods. In the former method an expectation measure, which is calculated by averaging a representative set of neighbouring solutions, is optimised instead of the main objective function. In the latter method, however, the main objective functions should be optimised with an additional constraint (variance measure) which restricts the optimisation process in terms of robustness of search agents.

In contrast to other branches of multi-objective optimisation, unfortunately, robust multi-objective optimisation has not gained deserved attention [2, 3]. As evidence, we conducted a publication report from 1994 to 2016 in ISI Web of Knowledge with the keywords "multi-objective optimisation" and "robust optimisation". We found that only about $0.5 \%$ of publications on these topics over the past decade contained both keywords. Among current works, there is a considerable number of studies that focused on robustness in single-objective search spaces (e.g. [4].) However, there should be more works in the literature on the investigation of robustness in multi-objective search spaces $[5,6]$.

In addition, the majority of current methods suffer from significant additional computational costs due to the need for additional function evaluations to confirm the robustness of solutions, making them impractical for solving real-world problems [7]. There are computationally expensive real-world problems that each objective function might take hours to run. Therefore, a designer cannot afford adding more sampled points. It is argued in the paper that surrogate-based techniques 
and archive-based approaches are two solutions to address this issue. However, the main drawback of surrogate techniques is the approximated models that introduce another level of uncertainty in this system.

Archive-based techniques alleviate this drawback by relying on the previously sampled points. They store the sampled points in a repository and use them to confirm the robustness of solutions. Their main advantage as compared to surrogate techniques is that they do not approximate the objective function since the sampled points are generated at some point during the optimisation process using true function evaluations. However, the drawback of this technique is the unreliability in finding enough number of points with high distribution within the neighbourhood of a solution due to the stochastic nature of stochastic optimisation algorithms.

The work described in this paper aims to address these issues, proposing a novel, computationally inexpensive approach for finding robust Pareto optimal solutions without additional sampling in multi-objective search spaces. In fact, a confidencebased method is proposed to quantify the confidence that we have when calculating the robustness measure. This makes the proposed confidence-based algorithms very practical since there is no need to calculate the objective value of sampled points. In a nutshell, a confidence measure assists a robust algorithm to make reliable decisions when comparing solutions using the previously sampled points.

The rest of this work is organised as follows: Section II provides the literature review, identifies the current gap in robust multi-objective optimisation, and outlines the contributions of this work. The confidence-based robust multi-objective optimisation is then proposed in Section III. The performance indicators, test functions, results, and discussion are provided in Section IV. Section V approximates the robust Pareto front for a marine propeller design problem to show the practicability of the approach proposed in solving real-world computationally expensive problems with unknown robust Pareto optimal front. Finally, Section VI concludes the work and suggests some directions for future studies.

\section{Background and related work}

\subsection{Multi-objective optimisation}

As its name implies, more than one objective function should be considered in multi-objective optimisation. Without loss of generality, this kind of optimisation is formulated as a minimisation problem as follows:

$$
\begin{gathered}
\text { Minimise : } \quad F(\vec{x})=\left\{f_{1}(\vec{x}), f_{2}(\vec{x}), \ldots, f_{o}(\vec{x})\right\} \\
\text { Subject to }: \quad g_{i}(\vec{x}) \geq 0, i=1,2, \ldots, m \\
h_{i}(\vec{x})=0, i=1,2, \ldots, p \\
l b_{i} \leq x_{i} \leq u b_{i}, i=1,2, \ldots, n
\end{gathered}
$$

where $g$ shows the inequality constraints, $h$ indicates the equality constraints, $o$ is the number of objective functions, $m$ is the number of inequality constraints, $p$ is the number of equality constraints, and $\left[l b_{i}, u b_{i}\right]$ are the boundaries of the $i$ - $t h$ variable.
In this kind of optimisation there is no unique answer as for single-objective optimisation. As outlined by Coello et al. [8], there is a set of solutions for a multi-objective problem called the Pareto optimal set. This set represents trade-offs between the objective functions (Pareto optimal solutions) as the solution for a multi-objective problem.

The mathematical definitions of Pareto optimality, Pareto optimal set, and Pareto optimal front are as follows [9, 10]:

Definition 2.1. (Pareto Dominance): Suppose that there are two vectors such as: $\vec{x}=\left(x_{1}, x_{2}, \ldots, x_{k}\right)$ and $\vec{y}=\left(y_{1}, y_{2}, \ldots, y_{k}\right)$. Vector $\vec{x}$ dominates vector $\vec{y}$ (denote as $\vec{x}<\vec{y}$ ) iff:

$$
\begin{gathered}
\forall i \in(1,2, \ldots, o) \\
{\left[f_{i}(\vec{x}) \leq f_{i}(\vec{y})\right] \wedge\left[\exists i \in 1,2, \ldots, o: f_{i}(\vec{x})<f_{i}(\vec{y})\right]}
\end{gathered}
$$

Note that $\leq$ and $<$ convert to $\geq$ and $>$, respectively, in maximisation problems.

Definition 2.2. (Pareto Optimality): A solution $\vec{x} \in X$ is called Pareto-optimal iff:

$$
\{\nexists \vec{y} \in X \mid \vec{y}<\vec{x}\}
$$

Definition 2.3. (Pareto optimal set): The set of all Paretooptimal solutions:

$$
P S:=\{\vec{x}, \vec{y} \in X \mid \nexists \vec{y}<\vec{x}\}
$$

Definition 2.4. (Pareto optimal front): A set containing the value of objective functions for Pareto solutions set:

$$
\begin{gathered}
\forall i \in(1,2, \ldots, o) \\
P F:=\left\{f_{i}(\vec{x}) \mid \vec{x} \in P S\right\}
\end{gathered}
$$

The ultimate goal of multi-objective optimisation algorithms is to find the Pareto optimal solutions with the highest diversity [11]. In this way, there would be a diverse range of design options available for decision makers.

Before the proposal of a posteriori multi-objective algorithms, the solution of multi-objective problems would generally have been undertaken by a priori aggregation of objectives into a single objective. However, it is known that this method has two main disadvantages: difficulties in finding proper weights, and approximating non-convex Pareto optimal fronts $[12,13,14]$. Also, these methods should be executed multiple times to accumulate the Pareto front because only one "best" solution is obtained from each run. According to Deb [15], the main advantage of a posteriori technique is that information about the search space is exchanged between the search agents, the information exchange yields rapid convergence toward the true front. Maintaining the multi-objective formulation of a problem also assists in obtaining the Pareto front in a single run and allows the exploration of the behaviour of the problems across a range of design parameters and operating conditions, but requires the use of more complex metaheuristics and a need to address conflicting objectives [16]. 


\subsection{Robust optimisation}

In the literature, robust optimisation mostly refers to considering uncertainties in parameters during optimisation. The approach presented in this work, however, with appropriate modification can be used for considering any type of uncertainties, as long as the uncertainty is reflected in the evaluation of the objective functions. According to Beyer and Sendhoff [17], there are four main categories of uncertainties in the real world: operating conditions, parameters, outputs, and constraints. This classification acknowledges that uncertainty may happen in any elements of a system.

The first type of uncertainties affect environmental conditions when a system is operating. Some examples might be temperature, speed, or angle of attack. The second type originates from manufacturing imprecisions where the defining parameters of a solution are (inadvertently) changed after finding the optimum. The third type of uncertainties generally arise from inaccuracies in producing the output of the system. Some examples are meta-modelling, simulators, and approximated models. The last uncertainty occurs in the constraints, in which the boundaries of the search space are varied but the system does not change proportionately. In this work, we concentrate on the second type of uncertainties.

Similarly to non-robust optimisation, the concept of robust optimal solutions is different in search spaces with single and multiple objectives. There is one robust solution for problems with one objective. However, a set of robust solutions should be found for a problem with multiple objectives. Robust multi-objective optimisation can be formulated as a minimisation problem as follows:

$$
\begin{gathered}
\text { Minimise : } \quad F(\vec{x}+\vec{\delta})=\left\{f_{1}(\vec{x}+\vec{\delta}), f_{2}(\vec{x}+\vec{\delta}), \ldots, f_{o}(\vec{x}+\vec{\delta})\right\} \\
\text { Subject to }: \quad g_{i}(\vec{x}+\vec{\delta}) \geq 0, i=1,2, \ldots, m \\
h_{i}(\vec{x}+\vec{\delta})=0, i=1,2, \ldots, p \\
l b_{i} \leq x_{i} \leq u b_{i}, i=1,2, \ldots, n
\end{gathered}
$$

where $\vec{x}$ shows the set of parameters, $\vec{\delta}$ is the uncertainty vector corresponding to each variable in $\vec{x}$, which is a stochastic (random) variable with a given (known or unknown) probability density function, $o$ is the number of objective functions, $m$ is the number of inequality constraints, $p$ is the number of quality constraints, $\left[l b_{i}, u b_{i}\right]$ are the boundaries of the $i$-th variable.

In robust multi-objective optimisation, the set of nondominated and robust solutions is called the robust Pareto optimal set and the corresponding set of objective values is called the robust Pareto optimal front. These two new sets are defined as follows [2]:

Definition 2.5. (Robust Pareto optimal set): The set of all solutions that are non-dominated considering the objectives, the robust measure, and the applied noise intensity.

The projection of the robust Pareto optimal set in the objective space is called the robust Pareto optimal front and defined as follows:

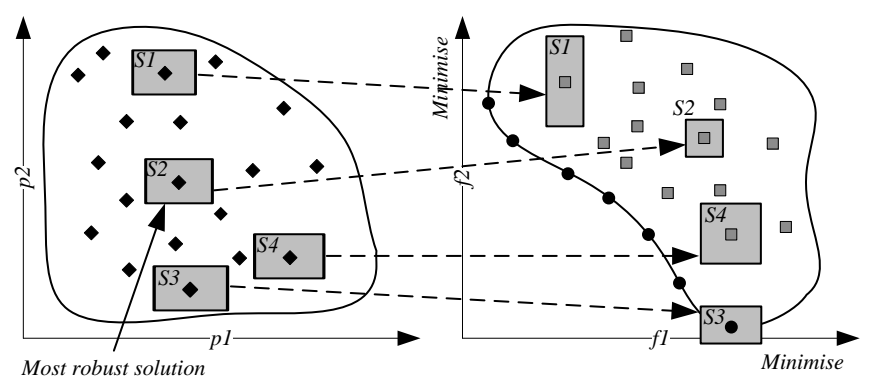

Figure 1: Effects of uncertainties in design parameters in a bi-objective search space
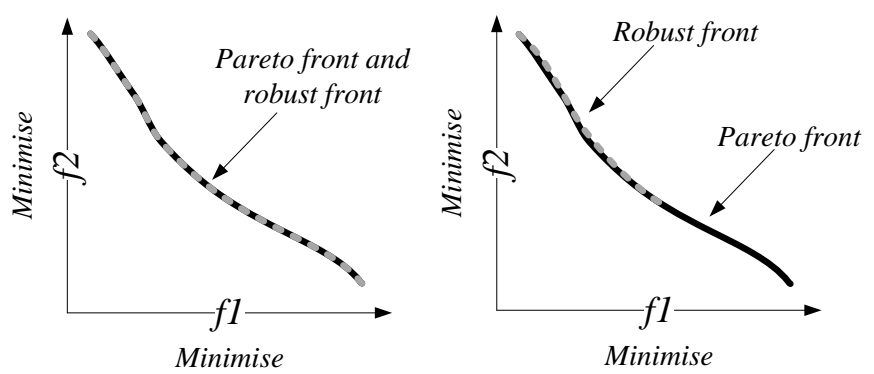

1

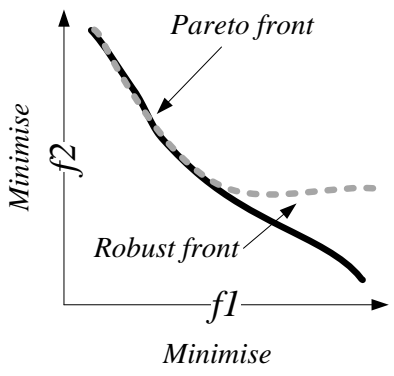

3

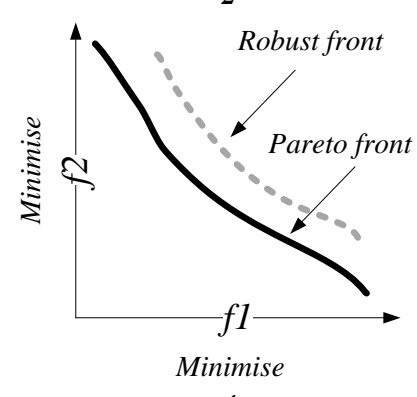

4

Figure 2: Four possible robust fronts compared to the Pareto front

Definition 2.6. (Robust Pareto optimal front): A set of objective vectors that are non-dominated considering the objectives, the robust measure, and the applied noise intensity.

The concept of robustness in a multi-objective search space is depicted in Figure 1. A mapping from a bi-parameter space to a bi-objective space is illustrated in this figure. It is evident that the same perturbation in the parameters $p 1$ and $p 2$ results in different variations in the objectives.

The most robust solution is $S 2$ and the worst one is $S 4$. As shown in Figure 2, there might be four types of robust Pareto fronts in multi-objective problems [18]: (a) the Pareto front is entirely made of robust solutions, (b) a portion of the front is made of robust solutions, (c) the robust front includes both dominated and non-dominated solutions, and (d) the robust Pareto front is dominated by the Pareto front.

\subsection{Related work and problem background}

In the literature, robust meta-heuristic optimisation is performed using two different measures: expectation and variance. Generally speaking, these measures are used for observing the 
behaviour in objective space in the neighbourhood of a particular solution to confirm robustness.

The mathematical formulation of robust optimisation using an expectation measure is presented in Equation 9.

$$
\text { Minimise : } \quad E(\vec{x})=\frac{1}{\left|B_{\delta}(\vec{x})\right|} \int_{\vec{y} \in B_{\delta}(\vec{x})} f(\vec{y}) d y
$$

where $B_{\delta}(\vec{x})$ shows the $\delta$-radius neighbourhood of the solution $\vec{x}$ and $\left|B_{\delta}(\vec{x})\right|$ indicates the hypervolume of the neighbourhood.

It may be inferred from Equation 9 that the expectation measure is the analytical integration of the main objective function over the maximum possible perturbation in the parameters. If the analytical integration of a multi-objective problem is unknown or difficult to obtain, the integration can be approximated by the Monte Carlo technique:

$$
E(\vec{x})=\frac{1}{H} \sum_{i=1}^{H} f\left(\vec{x}+\overrightarrow{\delta_{i}}\right)
$$

where $H$ is the number of samples.

Robust optimisation using expectation measures was named Type I robust optimisation by Deb and Gupta [1]. In this kind of optimisation, the objective function(s) are replaced by the expectation measure(s). Then, the expectation measure(s) are optimised.

Since the proposal of this kind of optimisation, many researchers proposed different expectation measures and tried to perform robust optimisation [19, 20, 21, 22, 23, 24]. Another way of robust optimisation using expectation measures is to consider them as separate objective functions [25, 26]. In this case the Pareto front finally obtained would indicate trade-offs between objective(s) and robustness. So the production for decision makers of a wide range of solutions with different degrees of robustness could be considered as the advantage of this method. As a drawback, however, considering expectation measures as additional objectives increases the computational complexity of a problem, as discussed by Brockhoff et al. [27, 28].

The second method of robust optimisation, using a variance measure, does not replace the main objective functions. An additional constraint is added to the problem in order to handle uncertainties. This constraint controls the variance of objectives of solutions in the objective space based on the local perturbations around the solution in the parameter space. Multiple random solutions is generated around the solutions in the parameter space and the variance of their corresponding objective values limited by a pre-defined threshold. A non-robust solution violates this constraint. Deb and Gupta named this method Type II robustness handling [1]. The mathematical expression is as follows [1]:

$$
\begin{gathered}
\text { Minimise : } f(\vec{x})=\left\{f_{1}(\vec{x}), f_{2}(\vec{x}), \ldots, f_{o}(\vec{x})\right\} \\
\text { Subject to : } V(\vec{x})=\frac{\|F(\vec{x})-f(\vec{x})\|}{\|f(\vec{x})\|} \leq \eta, \vec{x} \in S
\end{gathered}
$$

where $F(\vec{x})$ can be selected as the average or worst function value among the $H$ selected solutions, $\eta$ is a vector of thresholds in $[0,1]$, and $S$ indicates the feasible search space.
The robust solutions are favoured as $\eta$ decreases. There are also other variance measures proposed in the literature [5, 19].

In each of the above classes, several recent works have been done. In 2015, Dianz and Handl [29] applied an explicit and an implicit averaging strategy to real-world production planning problem due to the noisy fitness function (third type). It was found that implicit averaging method can be promising, but it should be accompanied with extra fitness evaluation to refine the final solutions obtained. To handle the same type of uncertainty, Fieldsend et al. proposed an Elite Accumulating Sampling (EAS) in 2015 and 2017 [30, 31, 32]. This technique regularly re-evaluates the elites and accumulate the evaluations, which results in the need for additional function evaluations and higher computational cost.

In 2016, Branke and Fei [33] proposed an efficient sampling when solving problems with uncertainties in variables (second type). This technique is based on a probability distance metric called Wasserstein distance that calculates the distance between a sample distribution and a target distribution. In other words, this method finds the best location to re-evaluate and accurately calculate the effective fitness for a solution. It also assigns weights to the samples in the area of disturbance for estimating the effective fitness. Despite the promising results on only three test functions, this method still requires additional function evaluations for each solution.

Another recent research was conducted by Meneghini et al. [34] in 2016, in which a robust algorithm was proposed without using expectation or variance measures. The authors changed the structure of MOEA/D in a way to use two populations: one population to find the Pareto optimal solutions and one population to confirm robustness by maximising the worst case scenario. The results of co-evolving populations were very promising, but increasing the number of function evaluation due to the use of two populations was the main shortcoming.

In 2017, Sabioni et al. [35] proposed an interactive robust optimisation framework to find desired robust solutions for problems with single or multiple objectives. The authors proposed a heuristic robust metric, which aims for finding robust solutions with a small sampling set. For sampling, they used the worst case scenario, which needs extra function evaluations. Note that the robustness measure was considered as an extra objective function to find trade-offs between the performance and robustness for decision makers. This increases the complexity of the optimisation process significantly as discussed by Brockho et al. [27, 28]. Also, the need for additional objective evaluation makes the framework less practical for computationally expensive real-world problems.

A recent attempt to reduce the number of function evaluations when using expectation measures was done by Cervantes et al. [36] in 2017. A dominance-based statistical testing was integrated to the selection operator of multi-objective genetic algorithms. Despite the merits of this technique in reducing the number of fitness evaluation while maintaining the quality of solutions, the method still requires function evaluations. Therefore, the computational cost is high compared to implicit averaging methods. Another similar work with the same drawbacks can be found in [37]. 
Zhou et al. [38] proposed a multi-objective robust algorithm based on Gaussian Process (GP), in which uncertainty is interpolated. This technique does not require extra function evaluation at all. However, using GP and interpolation makes it unreliable due the intrinsic errors in the interpolation process. In fact, this method can be considered as a meta-modal (surrogateassisted model) which suffers from the drawbacks mentioned above. Another similar interpolation-based meta-models were proposed in [39, 40, 41, 42].

In 2017 [43], Bayesian networks were employed to reduce the number of function evaluation required when using population-based algorithms. In this technique a Bayesian network is developed during the optimisation process as a memory of sampled points. In fact, the conditional dependencies between the best variables of the best solution obtained so-far is defined by the Bayesian network. The authors showed that this technique is beneficial in finding robust solutions. However, the solutions chosen by the Bayesian network would still have to be re-evaluated using an expectation measure to calculate their expected fitness.

An interesting recent research area aims to bridge the gap between robust optimisation and dynamic optimisation. In the field of dynamic optimisation, the optimal solutions change over time, so an algorithm needs to keep track of them. This can be achieved by investigating the changes in the neighbourhood of a solution in each iteration, which is similar to the process of confirming the robustness of a solution in robust optimisation. A seminal attempt to link these two areas is made in 2013 [44], in which a Robust Optimisation Over Time (ROOT) framework was proposed. In this framework, an optimisation algorithm finds an acceptable (optimal or sub-optimal) solution that changes slowly over time instead of tracking a moving global optimum. The similar concepts were used when solving multi-objective algorithms in 2017 as well [45].

In the field of crashworthiness design, there have been several works on robust optimisation as well to improve passenger safety. In 2011, for instance, Guangyong et. al. [46] proposed a multi-objective framework using several sigma criteria for measuring the variation of solutions in case of perturbation in parameters.

In 2013 [47], reliable and robust designs obtained for a typical vehicle structure subject to offset frontal crashing scenario were compared to deterministic approaches. It was observed that in case of uncertainties, reliable and robust fronts are both shifted away from their original place.

A robust design methodology was proposed in 2014 [48] using different sigma criteria to measure variations and find robust optimal solution for the problem of foam-filled thin-walled structure design. A Kriging model was used to create the surrogate (assisted with a sequential sampling method) of mean and standard deviations for different crashworthiness criteria

In 2017 [49], a multi-objective reliability-based design optimisation was proposed with the application in optimising a double-hat thin-walled structure. This was done by coupling with stamping uncertainties. The proposed technique allowed propagating uncertainties of material properties, process parameter, and resultant geometry from the foaming stage to crashing stage.

A multi-objective and multi-case reliability-based design optimisation (MOMCRBDO) was proposed in 2017 [50] to find reliable solutions for Tailor Rolled Blank (TRB), which is a technique to find light weight design while improving the crashworthiness of a car. It was observed that the proposed model is able to improve the reliability of Pareto optimal solutions and their robustness. A similar work can be found in [51, 52] as well.

In 2018 [53], a new multi-objective discrete robust algorithm was proposed. A successive Taguchi method was integrated to the algorithm and proved to be effective when solving benchmark and real-world problems (full scale vehicle structure for crashworthiness criteria).

Robust optimisation has been popular in other fields as well. In 2018, for instance, Du et. al. [54] minimised the impacts of uncertainness in the production event during a manufacturing process in the fashion industry. This was done by developing a Non-dominated Sorting Adaptive Differential Evolution (NSADE). The results showed the importance of considering uncertainties in production events, which can heavily impact the order scheduling in case of not handling it when optimising the problem.

\subsection{Problem statement}

The majority of current methods suffer from significant additional computational costs due to the need for additional function evaluations to confirm the robustness of solutions, making them impractical for solving real problems [7, 6]. There have been two main approaches proposed for reducing the computational costs (true function evaluation) of robustness handling methods as follows:

1. Archive-based methods, in which previously sampled solutions are saved and re-used during the optimisation in order to define the robustness of meta-heuristics' search agents [55, 56]

2. Surrogate-based techniques where a meta-model is employed to approximate the search space in the neighbourhood of solutions $[57,58]$

The second method is out of the scope of this work, so interested readers are referred to the comprehensive review by Jin [59]. The archive-based methods, which are the focus of this work, rely on previously evaluated solutions during robust optimisation.

The usefulness of archive-based methods was investigated and confirmed by a number of studies [56, 23, 60, 55]. They experimentally proved that previously sampled points could reduce the number of true function evaluations significantly and measure the robustness of solutions.

Although previously evaluated points have the potential to indicate the robustness of new solutions [56], the stochastic nature of meta-heuristics prevents this method from providing the highest reliability. In effect, the reliability of the archivebased methods is decreased as the number of archive members and true function evaluations are reduced. Deb et al. studied 


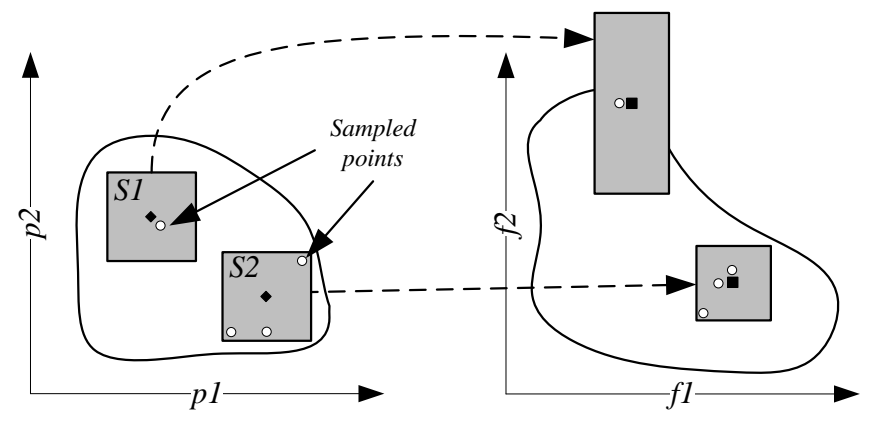

Figure 3: An example of the failure of archive-based methods in distinguishing a robust and non-robust solution. Note that the variances shown are the actual variances, not those detected by the sampling.

the effects of neighbourhood solutions in terms of finding the analytical robust front and found that finding the robust front becomes more challenging when the number of sampled solutions (or neighbouring solutions) decreases [18]. In addition, archive-based methods that only use previously sampled points are very unstable in the initial steps of optimisation due to the fewer number of sampled points.

All these reasons reduce the confidence of designers and decision makers in the performance of the archive-based methods and the quality of robust designs obtained. What makes these methods unreliable is: lack of sufficient previously sampled points in the archive, lack of a good distribution of sampled points around particular solutions, and lack of appropriate sampled solutions in a certain radius around particular solutions. One might think that the unreliability of the archive-based approaches is resolved after the initial steps of meta-heuristic optimisation, but the stochastic nature of meta-heuristics and the unknown shape of the search space prevent the archive-based methods from making confident decisions throughout the whole optimisation process.

Figure 3 shows an example of an archive-based method providing misleading information when relying on previously sampled points. In this figure the solution $S 2$ is more robust than $S 1$. In the archive, however, there is one sampled point for $S 1$ and three for $S 2$ to confirm the robustness. Since the sampled solution and $S 1$ are very close, the robustness measure indicates high robustness. However, the robustness measure for $S 2$ show less robustness due to the distribution of the solutions around $S 2$ in parameter and objective spaces. In this case, an archive-based method assumes that $S 1$ is more robust than $S 2$, while it is not. Such circumstances can happen throughout robust optimisation, which results in guiding the search agent of meta-heuristics toward misleading, non-robust regions of search spaces.

Therefore, it seems these robustness measures are not particularly reliable metrics for confirming the robustness of solutions when using previously sampled points. In multi-objective robust optimisation, the Pareto dominance based on robustness measure is also premature since there are possibilities that a non-robust solution dominates a robust solution only because of the unreliability of the archive-based methods. The reason for this unreliability of the robustness measures proposed so far is that the status of previously sampled points in parameter space is not considered. Only the magnitude of changes in the objective space are considered.

\subsection{Contributions}

To address the above-mentioned problem in robust singleobjective optimisation, we proposed a metric called the Confidence $(C)$ measure [61] to consider the status of the previously sampled points in a parameter space in order to improve the reliability of robustness measures. In this work, we propose a confidence-based Pareto optimality and dominance using both robustness and confidence metrics in order to make confident and reliable comparisons between solutions in a multi-objective search space. Also, we establish a novel approach of robust multi-objective optimisation using archive-based methods called Confidence-based Robust Multi-objective optimisation (CRMO). The proposed approach improves the reliability of archive-based methods without additional computational costs and assists designers to confidently rely on points previously evaluated during optimisation. In addition, the proposed approach allows designing different confidence-based methods for optimisation algorithms. To benchmark the proposed technique, some of our test functions [62, 63, 64] and performance indicators [65] will be used.

\section{Confidence-based Robust Multi-objective Optimisation}

There are three main factors that have impact on the quality of a robustness measure when using archive-based methods: the number of sampled points in the neighbourhood $(n)$, the radius of the neighbourhood $(r)$, and the distribution of the available points in the neighbourhood $(\sigma)$. These three main factors control the quality of the robustness metric, and the confidence that can be placed in its prediction; for this reason we named it the Confidence measure. The confidence measure is proposed as follows:

$$
\begin{gathered}
C=\frac{n}{r \cdot \sigma+1} \\
\sigma=\sqrt{\frac{\sum_{i=1}^{n}\left(\bar{d}-d_{i}\right)^{2}}{n-1}}
\end{gathered}
$$

where $n \geq 2, \bar{d}$ is the average of the distance between the current solution and all the sampled points within the neighbourhood, and $d_{i}$ is the Euclidean distance of the $i$-th sampled point to the current solution.

Note that when developing the confidence measure in the original paper [61], we had written an if-else statement to prevent the division by zero. However, in this work we devise a more general formula for $C$ and avoid division by zero by simply adding 1 to the sigma. Since this is a constant for all solutions, it does not impact the quality of the confidence measure and the performance of algorithms.

The proposed equation shows that the confidence measure is directly proportional to the number of sample points. This 
means that the greater the number of previously sampled points in the neighbourhood, the greater the confidence. In addition, the $C$ measure is inversely proportional to the radius of neighbourhood and the distribution of sampled points in it. Note that due to the stochastic nature of meta-heuristics, we assume that the distribution of sampled points within $r$ radius around a solution is approximately uniform. Therefore, if the sampled points are closer to the solution, they give better confidence about the robustness.

Since we have to calculate the Euclidean distance between the current solution and all the sampled points during optimisation, the computational complexity of the proposed metric is of $O\left(n_{s} D\right)$ where $n_{s}$ is the number of previously sampled points and $D$ indicates dimension. So the overall computational complexity is linear. It should be noted that the run time can also be decreased further by considering a limited number of recent sampled points during optimisation for long runs.

Some comments on the proposed $C$ metrics are:

- Confidence measure returns zero when there is no previously evaluated neighbouring solutions:

$$
n=0 \Longrightarrow C=0
$$

- Two solutions with the same number of neighbouring samples evaluated within equal radii are differentiated based on the dispersion:

$$
n_{1}=n_{2} \wedge r_{r}=r_{2} \wedge \sigma_{1}>\sigma_{2} \Longrightarrow C_{1}<C_{2}
$$

- Two solutions with an equal number of neighbouring solutions and similar distributions are differentiated based on the radii of their neighbourhoods.

$$
n_{1}=n_{2} \wedge \sigma_{1}=\sigma_{2} \wedge r_{1}>r_{2} \Longrightarrow C_{1}<C_{2}
$$

- The greater the value of $C$, the greater the level of confidence.

- The confidence measure can be extended to problems with any numbers of variables due to the use of Euclidean distance.

- The confidence measure is calculated based the status of neighbouring solutions without considering their objective values, so it is compatible with any kind of robustness measure.

- The computational complexity is of $O\left(n_{s} d\right)$, so it is computationally cheap to calculate.

- Confidence measure is easy to implement.

With the Confidence measure, the concepts of Pareto dominance, Pareto optimality, Pareto solution set, and Pareto front for robust optimisation can now be modified as follows (please note that the term robustness indicator $(R)$ is utilised to refer to any kind of robustness measure. So, $R(\vec{x})$ and $C(\vec{x})$ calculates the robustness and confidence of the solution $\vec{x}$ respectively.
Note that $f_{i}(\vec{x})$ shows the nominal values and $R_{i}(\vec{x})$ is the robust measure. The focus of the paper is on robust optimisation using expectation measures, in which the robustness measure is replaced with the objective function and optimised by a robust algorithm. Therefore, in the following definitions, $R_{i}(\vec{x})$ is used to compare two solutions.

Definition 3.1. (Confidence-based Pareto Dominance for minimisation): Suppose that there are two vectors: $\vec{x}=$ $\left(x_{1}, x_{2}, \ldots, x_{k}\right)$ and $\vec{y}=\left(y_{1}, y_{2}, \ldots, y_{k}\right)$.

Vector $\vec{x}$ confidently dominates vector $\vec{y}$ (denoted as $\vec{x} \prec_{c} \vec{y}$ ) if and only if:

$$
\begin{aligned}
& \forall i \in(1,2, \ldots, o) \\
& {\left[R_{i}(\vec{x}) \leq R_{i}(\vec{y})\right] \wedge[} {\left[\exists i \in(1,2, \ldots, o): R_{i}(\vec{x})<R_{i}(\vec{y})\right] } \\
& \wedge {[C(\vec{x}) \geq C(\vec{y})] }
\end{aligned}
$$

Note that confidence-based Pareto dominance in a maximisation problem can be achieved by converting $\leq$ and $<$ to $\geq$ and $>$. Since the concept of confidence measure does not change in minimisation and maximisation problems, the expression $[C(\vec{x}) \geq C(\vec{y})]$ is identical in both types of problems.

Definition 3.2. (Confidence-based Pareto Optimality): A solution $\vec{x} \in X$ is called confidence-based Pareto optimal if and only if:

$$
\left\{\nexists \vec{y} \in X \mid \vec{y} \prec_{c} \vec{x}\right\}
$$

Definition 3.3. (Confidence-based Pareto set): The set of all confidence-based Pareto optimal solutions:

$$
C P S=\left\{\vec{x} \in X \mid \nexists \vec{y} \in X, \vec{y} \prec_{c} \vec{x}\right\}
$$

Definition 3.4. (Confidence-based Pareto front): The set containing the value of objective functions for confidence-based Pareto solutions:

$$
\begin{gathered}
\forall i \in(1,2, \ldots, o) \\
C P F=\left\{R_{i}(\vec{x}) \mid \vec{x} \in C P_{s}\right\}
\end{gathered}
$$

Some comments on the proposed Pareto optimality concepts are:

- A solution is not able to confidently dominate another if it has less confidence:

$$
\vec{x}<\vec{y} \wedge C(\vec{x})<C(\vec{y}) \Longrightarrow \vec{x} \nprec_{c} \vec{y}
$$

- If the confidence of a solution is greater than or equal to another, the confidence-based Pareto dominance become equivalent to the normal Pareto dominance for that particular solution:

$$
C(\vec{x}) \geq C(\vec{y}) \Longrightarrow<_{c} \equiv<
$$

- If two solutions are non-dominated with respect to each other, they are also confidently non-dominated with respect to each other:

$$
\vec{x} \nprec \vec{y} \wedge \vec{y} \nprec \vec{x} \Longrightarrow\left(\vec{x} \nprec_{c} \vec{y}\right) \wedge\left(\vec{y} \nprec_{c} \vec{x}\right)
$$


- The confidence-based Pareto solution set contains all the confident solutions and none of them can confidently dominate another.

The proposed confidence-based Pareto optimality, dominance, set, and front can be integrated with different metaheuristics. In other words, various combinations of these confidence-based concepts can be integrated into the modules of multi-objective optimisation algorithms in order to perform a reliable robust optimisation. As mentioned above, this type of robust optimisation is named CRMO.

As the case study, the MOPSO algorithm is employed in this work. Confidence-based Pareto dominance is integrated with the MOPSO algorithm in order to allow it to perform confidence-based robust multi-objective optimisation. The archive controller module of MOPSO is targeted as the main tool for integration. In the MOPSO algorithm, the archive controller module handles insertion or deletion of solutions in the archive. In the Confidence-based Robust MOPSO (CRMOPSO), the following rules are proposed to provide a confidence-based archive controller:

- If a new solution is confidently dominated by one of the archive member it should be omitted immediately.

- If the new solution is not confidently dominated by the archive members, it should be added to the archive.

- If a member of the archive is confidently dominated by a new solution, it is removed.

- If the archive is full the adaptive grid mechanism is triggered.

There is also a modification to the archive itself in order to maintain the reliability of the archive. We require CRMOPSO to update the confidence level of archive members at each iteration. This mechanism allows archive members to improve their confidence level using the current status of the previously sampled solutions. In this case, an archive member may be omitted if its confidence level does not improve over the iterations. Note that this method should be employed when saving and using all the previously sampled points. If CRMOPSO only utilises a set of recent previously sampled points, this method would not be effective since the highly confident robust solutions will be prone also to be omitted from the archive. Note that the previously sampled points are the visited solutions by all particles during optimisation. Implicit averaging methods rely on the algorithm itself using these sampled points to decide about the robustness of solutions.

It also may be noticed that the dominance of a solution in CRMOPSO is identical to that of RMOPSO, and the confidencebased dominance is only applied to the archive controller. This prevents CRMOPSO from showing degraded exploration.

The detailed procedure of the proposed CRMOPSO is shown in Figure 4. As may be seen in this figure, CRMOPSO starts with a random population. This population is evaluated using the expectation measure. The position and robustness value (expected/effective fitness) of each particle in the first population are stored in the repository of sampled points. At this stage, all the non-dominated solutions are found using a regular Pareto optimal dominate to be inserted in the archive. The confidence level of each archive member is calculated. The CRMOPSO algorithm then repeatedly performs the followings steps until the satisfaction of the end criterion:

To update the position of a particle, a solution is selected as GBEST. The velocity and position vectors are then calculated. The particle is evaluated using the robustness measure. The PBEST is updated using the regular Pareto optimal dominance, while the particle is inserted into the archive considering the confidence level (using the proposed confidence-based Pareto dominance operator) and the rules mentioned above. The position and effective fitness of the particle are then stored in the repository of sampled points. After updating the position of each particle, the archive is updated to remove all the nondominated solutions. At this stage, the confidence level of all archive members is re-calculated based on all the sampled points in the disturbance area. Finally, the archive is returns as the final answer.

\section{Computational experiments and discussion}

\subsection{Test Problems}

There appear to be few test functions in the literature for benchmarking robust multi-objective algorithms. Two sets of test problems proposed by Deb and Gupta [1] and GasparCunha et al. [6] can be considered as the only test problems in this field. The test functions of Deb and Gupta [1] mimicked the four possible situations of robust front compared to true front (shown in Figure 2). The test functions of GasparCunha et al. [6] mostly have regions with different degree of robustness. Although these two sets of test functions are able to provide different types of robust Pareto optimal fronts, there are other issues in robust optimisation of real problems such as discontinuous robust/global Pareto optimal fronts, convex/concave robust/global Pareto optimal fronts, and multi-modality. Therefore, we have proposed challenging test functions in [62] to fill this gap and utilize them in this study. The test functions are generated with similar frameworks to that we proposed in [62].

The shapes of parameter spaces and objective spaces that can be constructed using the frameworks are illustrated in Figure 5 and Figure 6. Figure 5 shows how the first framework allows us to generate any desired shape and robustness for local and global fronts. Figure 6 shows how the second framework generates multi-modal parameter spaces and objective spaces including disconnected regions with different robustness. The last local front is the most robust front, so this framework is able to provide very challenging and deceptive test beds.

We employed both frameworks to generate 6 test functions in order to be able to compare the proposed method of robust multi-objective optimisation with other methods. A summary of all 10 test functions is provided in TABLE 1.

The shape of the test functions can be seen in Figure 9, Figure 10 and Figure 11. These figures show that this set of test problems provides different robust front types: convex, nonconvex, discontinuous, and multi-modal. Due to the simplicity 


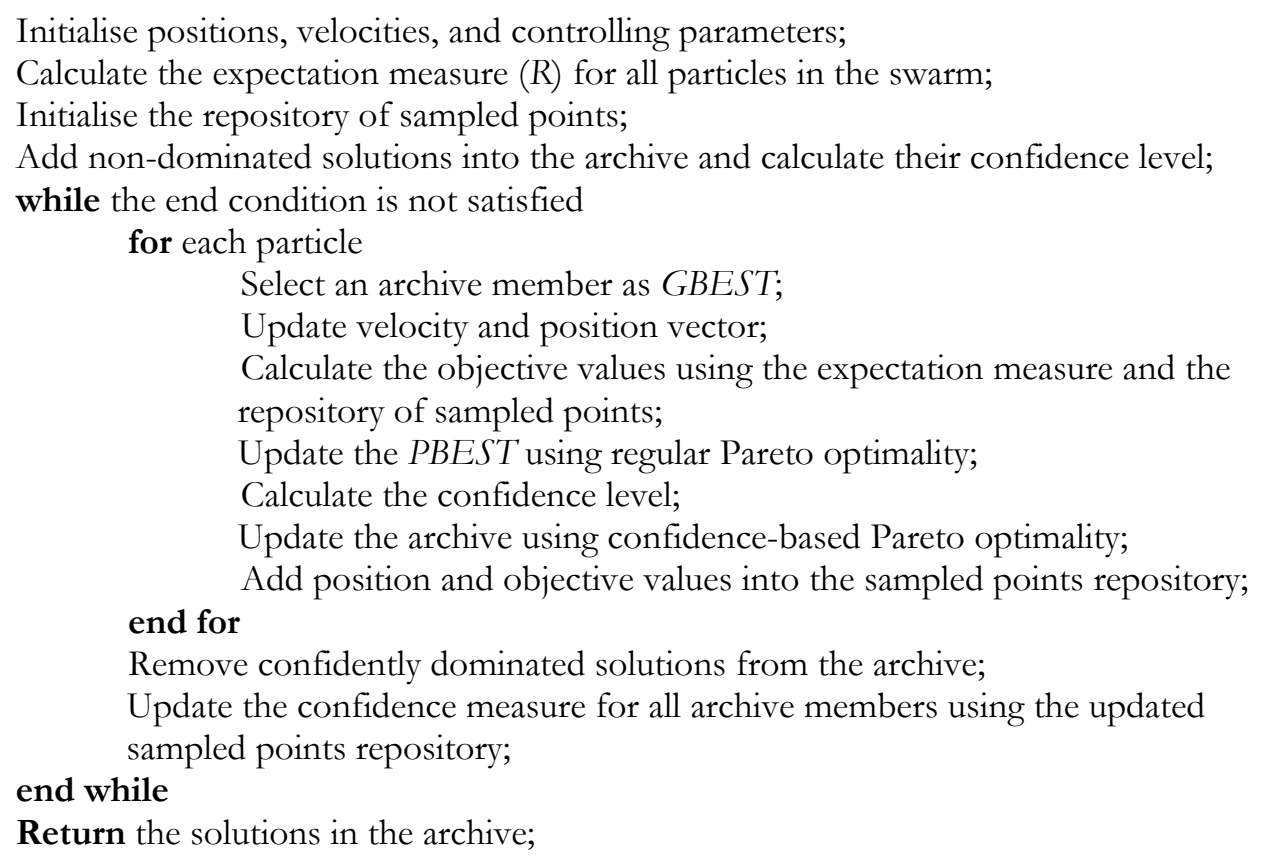

Figure 4: Pseudocode of CRMOPSO.

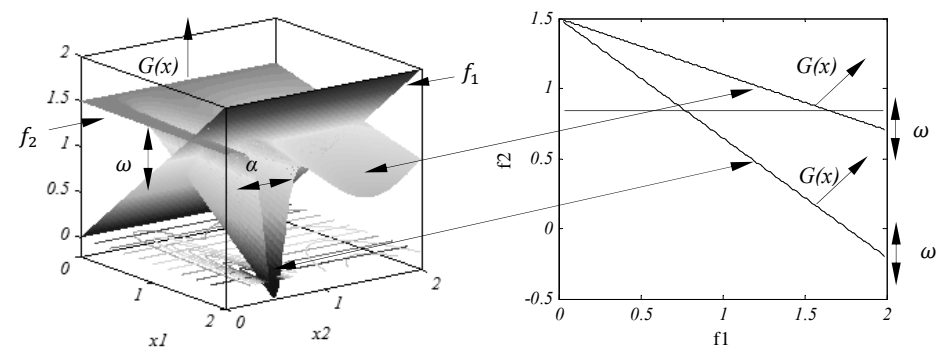

Figure 5: Framework 1 for generating test functions.

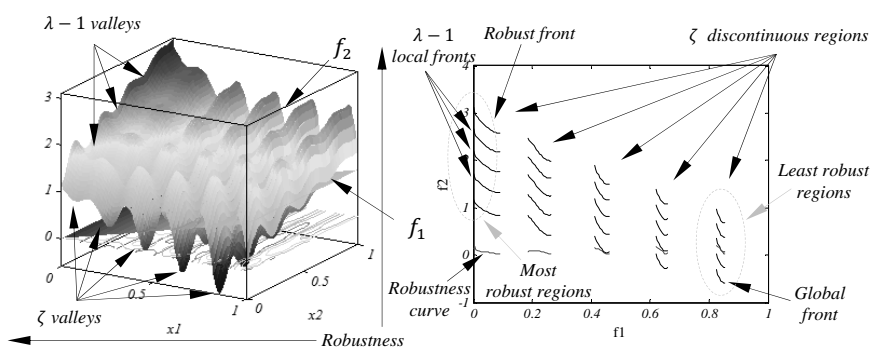

Figure 6: Framework 2 for generating test functions.

and uni-modal nature, MTP1-MTP3 can benchmark the convergence of the algorithms. In addition, the performance of algorithms can be benchmarked by MTP4-MTP8 in terms of coverage and adaptability to robust fronts with different shapes. Finally, MTP9 and MTP10 are able to test the performance of robust meta-heuristics in terms of avoiding non-robust local fronts and approximating robust fronts with disconnected regions. Note that the (inverse of) robustness of the robust fronts is indicated by the overlaid grey lines in Figure 9, Figure 10
Table 1: Test functions utilised in this work

\begin{tabular}{l|l}
\hline Name & Details \\
\hline MTP1 & Deb and Gupta's first test function [1] \\
\hline MTP2 & TP3 by Gaspar-Cunha et al. $[6]$ \\
\hline MTP3 & TP4 by Gaspar-Cunha et al. [6] \\
\hline MTP4 & Step-shaped Pareto front \\
\hline MTP5 & Generated by framework $1: \alpha=0.1, \beta 1=, \beta 2=0.5$ \\
\hline MTP6 & Generated by framework 1: $\alpha=0.1, \beta 1=1, \beta 2=0.5$ \\
\hline MTP7 & Generated by framework 1: $\alpha=0.1, \beta 1=1, \beta 2=1.5$ \\
\hline MTP8 & Generated by framework $1: \alpha=0.1, \beta 1=0.5, \beta 2=1.5$ \\
\hline MTP9 & Generated by framework $2: \zeta=4, \lambda=6$ \\
\hline MTP10 & Generated by framework $2: \zeta=8, \lambda=6$ \\
\hline
\end{tabular}

and Figure 11 (i.e. low values are preferred). The grey lines are cumulative value for the robustness of $f 1$ and $f 2$ and that it is plotted for given values of $f 1$ (i.e. a point on the Pareto front has its robustness plotted at the same $x$ position).

\subsection{Performance Measures}

In the literature there are only three specific performance indicators for robust multi-objective meta-heuristics proposed by the authors in 2015 [65]. This is the main reason why all of the works in this field qualitatively evaluate and compare different algorithms based on the shape of the robust Pareto optimal fronts obtained, as can be seen in a number of articles $[55,1,6,18,20,21,66,67]$. In contrast, there have been many performance measures for multi-objective metaheuristics [68]. The performance indicators mostly measure two important factors in multi-objective optimisation: convergence toward the true front, and the diversity of the Pareto optimal solutions obtained. The current performance measures can 


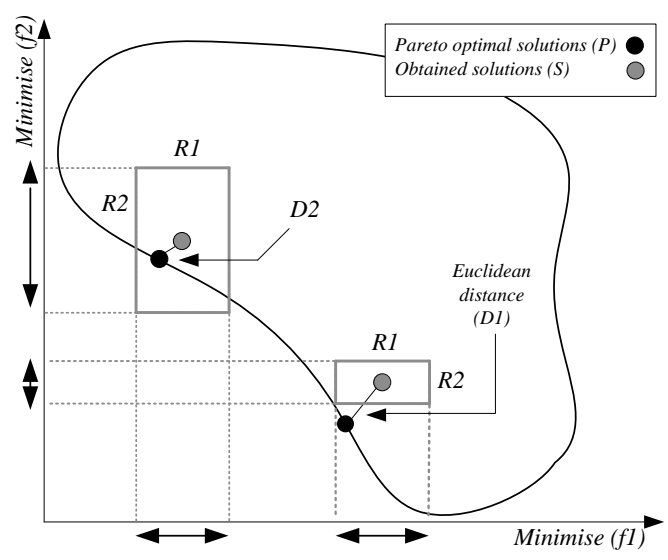

Figure 7: General features of the proposed robust convergence metric.

be classified into three main categories: 1 - convergence of the solutions, 2- spread of the solutions, and 3- a combination of convergence and spread of solutions. The first two classifications are more reliable than the latter since the combination of these measures cannot reliably show superiority between two set of Pareto optimal solutions [68]. The three performance measures that we have proposed in [65] and employ in this work are presented in the following paragraphs.

The first measure is a convergence metric $(\Xi)$ that involves the robustness of solutions in order to favour solutions obtained in the robust regions of the front and defined as follows:

$$
\begin{gathered}
\Xi=\frac{1}{N} \sum_{i=1}^{N} \xi_{i} \\
\xi_{i}=d\left(\vec{x}, P_{n}\right) * \sum_{i=1}^{O} R_{i}(\vec{x})
\end{gathered}
$$

where $P_{n}$ is the closest true Pareto optimal solution to $\vec{x}, d()$ calculates the Euclidean distance, $N$ is the number of solutions obtained, $O$ indicates the number of objectives, and $R_{i}(\vec{x})$ is the robustness of $\vec{x}$ on the $i$-th objective.

A conceptual model of the proposed metric is shown in Figure 7. It may be observed that this metric considers the robustness of the solution obtained in addition to its Euclidean distance to the closest reference point in the main Pareto optimal front.

Note that in order to implement $\Xi$, the Pareto optimal front and robustness should be known and this metric shows higher convergence as the solutions obtained get closer to the robust reference points.

The second metric $(\Phi)$ is a coverage metric inspired by Lewis et al. [69]. As shown in Fig. 8, the objective space is divided into several sectors. The sectors can be robust or non-robust. This metric calculates the ratio of robust and non-robust sectors as follows:

$$
\begin{gathered}
\Phi=\frac{1}{N} \sum_{n=1}^{n} \phi_{n} \\
\phi_{n}= \begin{cases}1 & \exists \vec{x} \in P S, \alpha_{n-1} \leq \tan \frac{f_{1}(\vec{x})}{f_{2}(\vec{x})} \leq \alpha_{n}, R\left(P_{n}\right) \leq R_{\text {min }} \\
0 & \text { otherwise }\end{cases}
\end{gathered}
$$

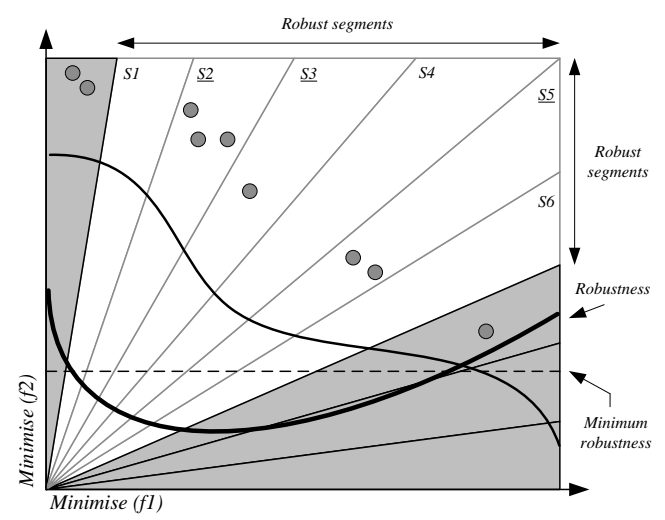

Figure 8: General features of the proposed robustness coverage metric.

where $N$ is the number of robust sectors, $\vec{x}$ is an approximation of the Pareto optimal solutions obtained, $\alpha_{n}$ is the angle of the right line of a segment, $P_{n}$ indicates the closet true Pareto optimal solution to $\vec{x}$, and $R_{\text {min }}$ is a minimum robustness value defined by the user. Note that there should be an exception when there is no robust segment in order to prevent division by zero.

The third metric is a robust success ratio $(\Gamma)$ that calculates the ratio of robust and non-robust solutions and defined as follows:

$$
\begin{gathered}
\Gamma=\frac{\gamma^{R}}{1+\gamma^{N} R}, \gamma^{R}=\sum_{i=1}^{M} p_{i}^{R}, \gamma^{N} R=\sum_{i=1}^{M} p_{i}^{N R} \\
p_{m}^{R}= \begin{cases}1 & \exists \vec{x} \in P S, \beta_{m-1} \leq f_{1}(\vec{x}) \leq \beta_{m}, R\left(P_{n}\right) \leq R_{\text {min }} \\
0 & \text { otherwise }\end{cases} \\
p_{m}^{N R}= \begin{cases}1 & \exists \vec{x} \in P S, \beta_{m-1} \leq f_{1}(\vec{x}) \leq \beta_{m}, R\left(P_{n}\right)>R_{m i n} \\
0 & \text { otherwise }\end{cases}
\end{gathered}
$$

where $M$ is the number of Pareto optimal solutions obtained, $\vec{x}$ is an approximation of the Pareto optima solutions obtained, $\beta_{n}$ is the $f_{1}$ value of the right line of a segment, $P_{n}$ is the closet true Pareto optimal solution to $\vec{x}$, and $R_{\text {min }}$ is the minimum robustness value.

With this set of performance measures $(\Xi, \Phi, \Gamma)$ the performance of different robust multi-objective algorithms employed in this work can be quantified.

\subsection{Experimental set-up}

We implemented three versions of Robust MOPSO: an Explicit averaging Robust MOPSO (ERMOPSO), Implicit averaging Robust MOPSO (IRMOPSO), and Confidence-based Robust MOPSO (CRMOPSO). The method of explicit averaging in ERMOPSO is identical to that of RNSGA-II proposed by Deb and Gupta [1]. In this method $H$ number of sampled solutions is created by the Latin Hypercube Sampling method around every candidate solution to investigate and confirm robustness during optimisation. This provides the highest reliability and is helpful for verifying the performance of the proposed algorithm as the most reliable reference. It does, however, markedly increase the computational load per iteration. In 
Table 2: Statistical results of CRMOPSO, IRMOPSO, and ERMOPSO on the test functions

\begin{tabular}{|c|c|c|c|c|c|c|c|c|c|}
\hline & \multicolumn{3}{|c|}{ CRMOPSO } & \multicolumn{3}{|c|}{ IRMOPSO } & \multicolumn{3}{|c|}{ ERMOPSO } \\
\hline & $\Xi$ & $\Phi$ & $\Gamma$ & $\Xi$ & $\Phi$ & $\Gamma$ & $\Xi$ & $\Phi$ & $\Gamma$ \\
\hline MTP1 & $0.27 \pm 0.14$ & $0.30 \pm 0.09$ & $42.2 \pm 16.6$ & $0.10 \pm 0.08$ & $0.56 \pm 0.05$ & $6.16 \pm 7.41$ & $2.33 \pm 1.8$ & $0.17 \pm 0.17$ & $9.22 \pm 5.76$ \\
\hline MTP2 & $0.01 \pm 0.01$ & $0.29 \pm 0.09$ & $1.69 \pm 0.99$ & $0.01 \pm 0.03$ & $0.19 \pm 0.05$ & $0.92 \pm 0.53$ & $0.00 \pm 0.00$ & $0.42 \pm 0.08$ & $0.67 \pm 0.34$ \\
\hline MTP3 & $0.02 \pm 0.01$ & $0.46 \pm 0.14$ & $0.75 \pm 0.27$ & $0.01 \pm 0.01$ & $0.38 \pm 0.12$ & $0.38 \pm 0.14$ & $0.01 \pm 0.01$ & $0.51 \pm 0.16$ & $0.53 \pm 0.10$ \\
\hline MTP4 & $0.01 \pm 0.00$ & $0.53 \pm 0.21$ & $7.52 \pm 7.67$ & $0.01 \pm 0.00$ & $0.37 \pm 0.14$ & $0.45 \pm 0.42$ & $0.01 \pm 0.00$ & $0.54 \pm 0.17$ & $1.54 \pm 1.88$ \\
\hline MTP5 & $0.09 \pm 0.03$ & $0.32 \pm 0.08$ & $1.17 \pm 0.45$ & $0.10 \pm 0.03$ & $0.47 \pm 0.20$ & $2.38 \pm 1.84$ & $0.66 \pm 0.76$ & $0.46 \pm 0.21$ & $1.92 \pm 1.33$ \\
\hline MTP6 & $0.19 \pm 0.06$ & $0.57 \pm 0.11$ & $6.65 \pm 3.69$ & $0.28 \pm 0.05$ & $0.55 \pm 0.09$ & $8.12 \pm 2.29$ & $3.17 \pm 5.37$ & $0.39 \pm 0.31$ & $3.19 \pm 3.40$ \\
\hline MTP7 & $0.19 \pm 0.08$ & $0.53 \pm 0.20$ & $9.32 \pm 18.2$ & $0.25 \pm 0.08$ & $0.46 \pm 0.13$ & $3.75 \pm 10.4$ & $0.64 \pm 0.95$ & $0.58 \pm 0.19$ & $1.21 \pm 1.45$ \\
\hline MTP8 & $0.21 \pm 0.03$ & $0.84 \pm 0.07$ & $3.64 \pm 2.46$ & $0.22 \pm 0.03$ & $0.63 \pm 0.15$ & $6.32 \pm 16.6$ & $0.77 \pm 1.07$ & $0.70 \pm 0.21$ & $2.14 \pm 1.89$ \\
\hline MTP9 & $0.02 \pm 0.00$ & $0.54 \pm 0.17$ & $15.7 \pm 10.0$ & $0.022 \pm 0.00$ & $0.33 \pm 0.09$ & $14.8 \pm 7.95$ & $0.023 \pm 0.00$ & $0.47 \pm 0.13$ & $17.9 \pm 11.4$ \\
\hline MTP10 & $0.02 \pm 0.00$ & $0.59 \pm 0.12$ & $39.5 \pm 20.6$ & $0.03 \pm 0.00$ & $0.45 \pm 0.07$ & $28.3 \pm 8.91$ & $0.03 \pm 0.00$ & $0.63 \pm 0.14$ & $44.9 \pm 19.07$ \\
\hline
\end{tabular}

contrast, IRMOPSO utilises the previously sampled solutions to confirm robustness. In this case, robustness of a candidate solution is evaluated based on the available solutions in the neighbourhood during optimisation. We save all the sampled solutions during optimisation, in a manner similar to Branke [56] and Dippel [60]. As was discussed in the motivation for this work, IRMOPSO shows less reliability and its use will allow comparison of CRMOPSO in terms of improved reliability. Robustness is calculated by an expectation measure with simple averaging of the neighbouring solutions for all algorithms.

In order to provide a fair comparison and see if the proposed method is reliable and effective, we used the same number of function evaluations $(100,000)$ for each of the algorithms. The number of trial solutions in the population was 100 for all algorithms, so the maximum number of iterations for IRMOPSO and CRMOPSO was 1000. Since ERMOPSO uses explicit averaging, however, we use 4 re-sampling points and 250 iterations in order to achieve the total number of 100,000 function evaluations. Another assumption was $10 \%$ fluctuation in the parameters to simulate uncertainties in parameters.

Each algorithm was run 30 times and the results of performance metrics are reported in TABLE 2. Note the quantitative results are presented in the form of (average \pm standard deviation). For the performance measures, $10 \%$ noise was considered and $R_{\min }$ was calculated using the equation $\min _{\text {robustness }}+\left(\right.$ max $\left._{\text {robustness }}-\min _{\text {robustness }} \times 0.1\right)$ where min $_{\text {robustness }}$ is the minimum of the robustness curve and max $_{\text {robustness }}$ indicates the maximum of robustness in the test functions. In addition, the best robust fronts obtained by each algorithm are illustrated in Figure 9, Figure 10 and Figure 11. We did not compare the results with other meta-heuristics since the different mechanisms of the algorithms would prevent us from distinguishing whether the superior results of one algorithm were due to CRMO or the algorithm's underlying mechanism.

\subsection{Discussion of results}

It may be observed in Figure 9 that the most well-distributed robust front for MTP1 is that of IRMOPSO. This is because the robust front is identical to the main Pareto front (the first case shown in Figure 2). Since IRMOPSO uses an implicit averaging method, the convergence is greater than for other al- gorithms. However, the greatest density of solutions is in the middle of the robust front, which has less robustness compared to the left end of the main front. Figure 9 shows that the robust front obtained by CRMOPSO for MTP1 is slightly more widely distributed than that of ERMOPSO. In addition, a high density of solutions can be observed on the left end of the robust font. Despite the apparent similarity of CRMOPSO and ERMOPSO in Figure 9, Table 2 shows that the CRMOPSO algorithm provided much better statistical results compared to ERMOPSO. This shows that the confidence-based Pareto dominance operators allow CRMOPSO to provide more reliable performance within an equal number of function evaluations. The statistical results of IRMOPSO for this test problem were slightly better that CRMOPSO, in terms of convergence, due to the nature of MTP1. However, the results show that the confidence-based operators were ably to provide high reliability without significant negative impact on convergence.

The results for MTP2 are slightly different to those of MTP1, for which ERMOPSO showed the highest convergence. Figure 9 shows that ERMOPSO approximated the entire Pareto optimal front and there was no tendency to favour robust regions of the front. This resulted in this algorithm showing the worst coverage and success ratio on MTP2. In contrast, CRMOPSO and IRMOPSO found better approximations of the robust front. The solutions obtained by CRMOPSO were clustered on the left side of the robust front and it seems there is resistance to generating any solutions on the less robust regions of the Pareto optimal front. Although the robust Pareto optimal solutions found by IRMOPSO followed a similar pattern, some of them are located on the least robust regions of the front.

The MTP3 test function has three separate robust regions. The robustness curve shows that the robust regions are around $f 1=0,0.4,1$. The results in TABLE 2 demonstrate that the convergence of CRMOPSO was again slightly worse than IRMOPSO and ERMOPSO. However, CRMOPSO showed superior coverage and success ratio. The shape of the approximated robust Pareto front in Figure 9 shows that ERMOPSO behaved similarly on this test function, in contrast to MTP2 where there was no particular tendency to favour robust regions. A slight bias to robust regions can be observed in the robust solutions found by IRMOPSO. The approximated robust Pareto optimal front of CRMOPSO, however, shows that this algorithm had a 


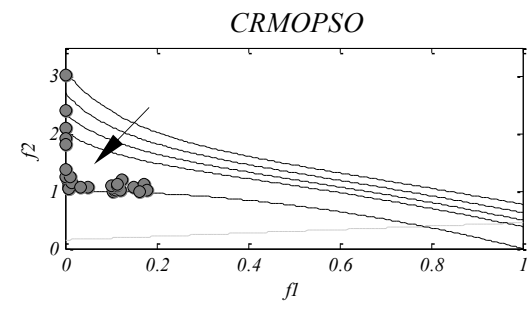

CRMOPSO

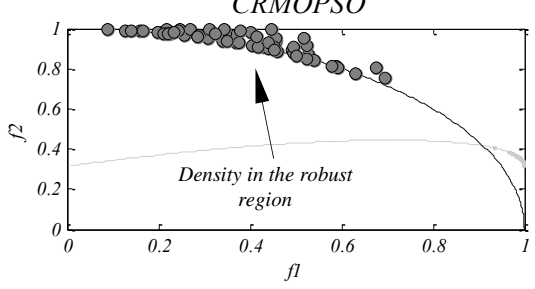

CRMOPSO

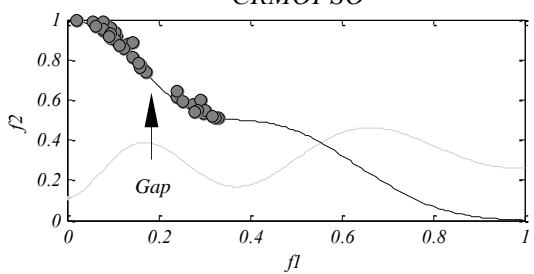

CRMOPSO

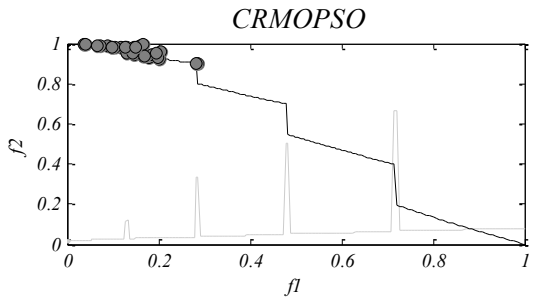

IRMOPSO

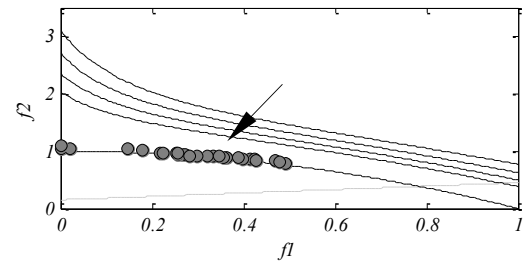

IRMOPSO

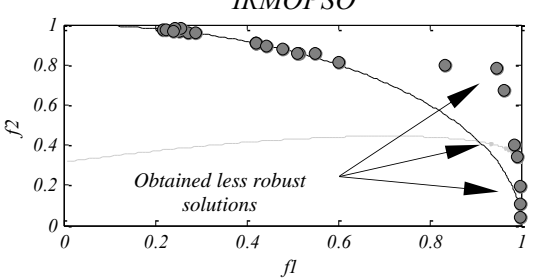

IRMOPSO

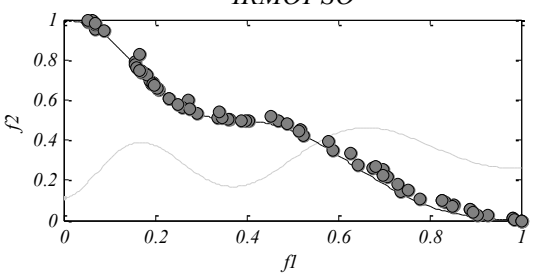

IRMOPSO

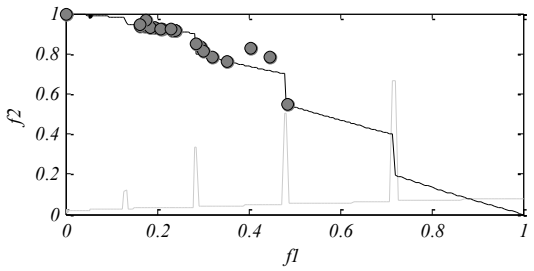

ERMOPSO

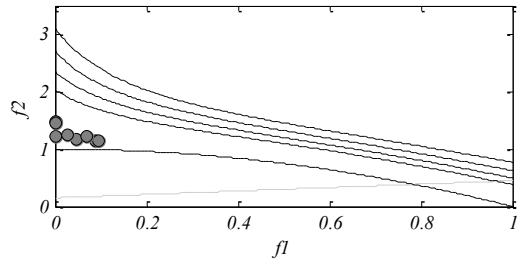

ERMOPSO

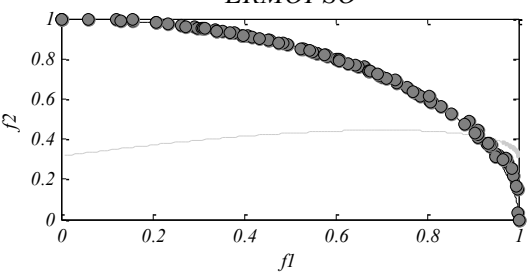

ERMOPSO

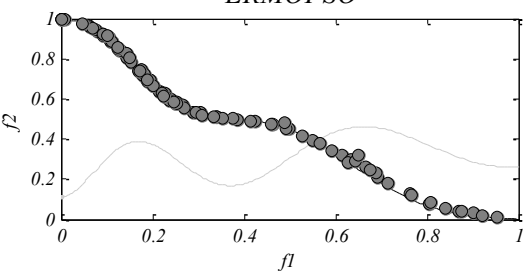

ERMOPSO

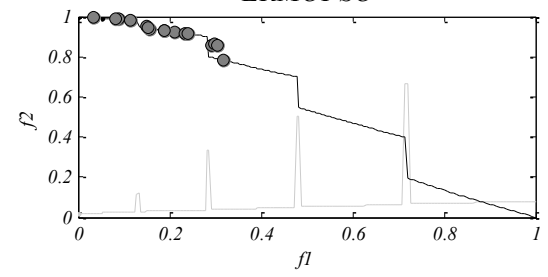

Figure 9: Robust fronts obtained for MTP1 to MTP4, one test case per row.

greater ability to guide its solutions toward robust regions of the Pareto optimal front. There is a gap between the solutions obtained on the least robust region.

The behaviour of algorithms in terms of finding robust regions of the Pareto optimal front can be observed more clearly with MTP4. In fact, we deliberately arranged this test function to have a stair-shaped Pareto optimal front to test the ability of algorithms in terms on converging toward robust regions of the front and refraining from finding non-robust solutions. The quantitative results of the algorithms on MTP4 show that the results of CRMOPSO are significantly better than IRMOPSO and ERMOPSO. The CRMOPSO algorithm only approximates the first two stairs, which are considered the most robust regions of the Pareto optimal front, as shown in Figure 9. However, the robust solutions obtained by IRMOPSO and ERMOPSO tend to be distributed on less robust regions as well.

Test functions MTP5 to MTP8 are bi-modal. The local front is the robust front, so the behaviour of algorithms in favouring a robust front instead of a global front can be investigated. In these test functions, robust and global fronts have different shapes in order to extensively test the performance of the algorithms. In MTP5 the shape of both local and global fronts is convex. In contrast to the quantitative results of MTP1 to MTP4, the CRMOPSO algorithm showed the highest conver- gence on this test function. This shows that although the convergence of the proposed method is slightly low, this may be favourable when solving multi-modal test functions. The coverage and success ratio of CRMOPSO were also very good on this test function. The best robust solutions obtained for all algorithms are again illustrated in Figure 10. It may be seen that the best front obtained was from CRMOPSO. This algorithm did not approximate even one non-robust solution for this test function, proving the merits of the proposed method. IRMOPSO was not able to approximate the robust front over 20 runs for this problem showing the potential unreliability of using previous samples without a confidence measure. The ERMOPSO algorithm also mostly tended to approximate the global front, but there were some solutions found on the robust front.

As Figure 10 shows, MTP6 and MTP7 have identical linear global fronts that make the approximation of the global front easy. We deliberately arranged these two test functions to have linear global fronts to observe what resistance the algorithms provided in avoiding non-robust but easily obtained fronts. MTP6 has a convex robust front, whereas MTP7 has a concave robust front. The approximated robust solutions in Figure 10 show that IRMOPSO again failed to estimate the robust Pareto optimal front on both test cases. This is due to the simplicity of the global front and unreliability of the IR- 

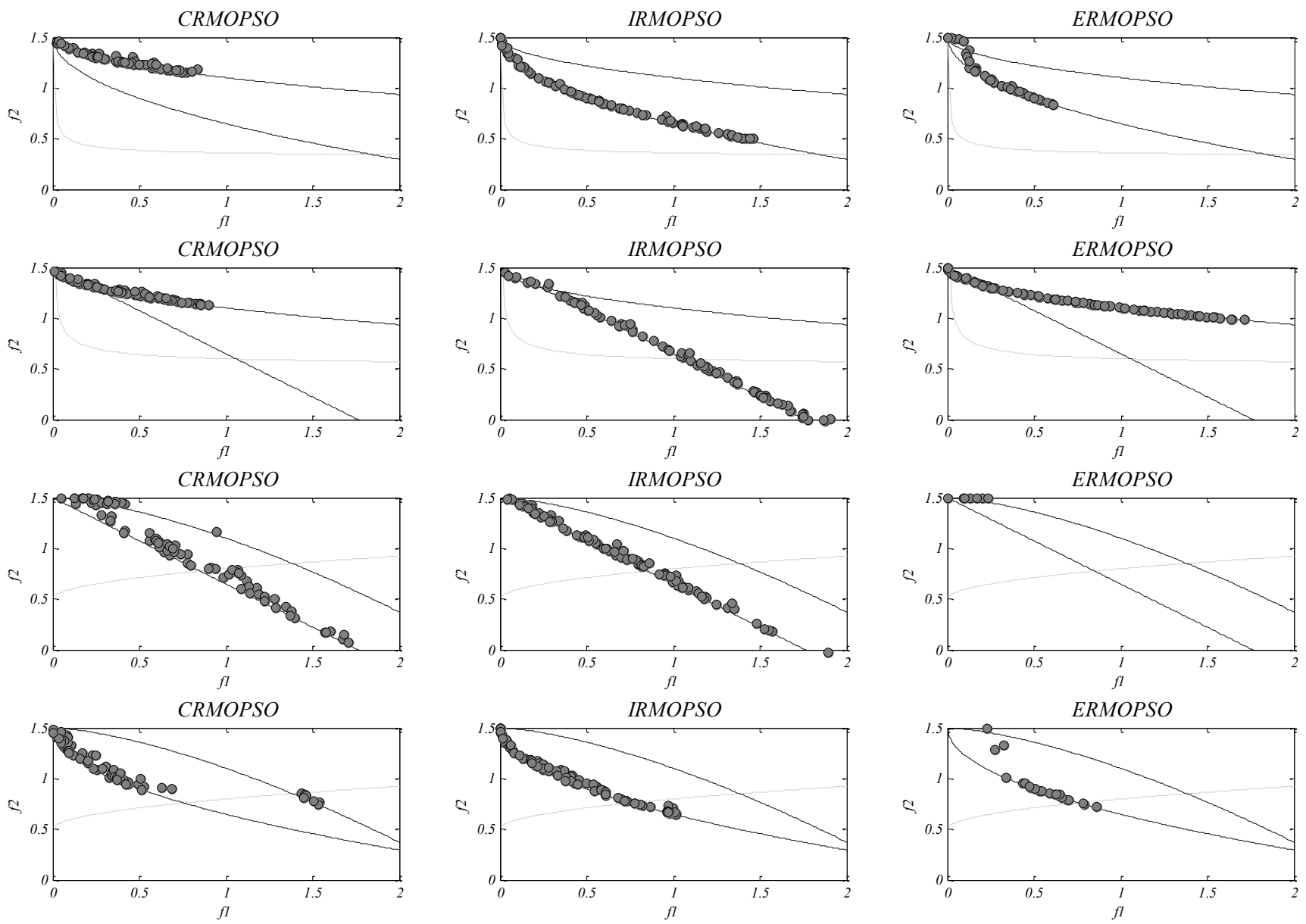

Figure 10: Robust fronts obtained for MTP5 to MTP8, one test case per row.
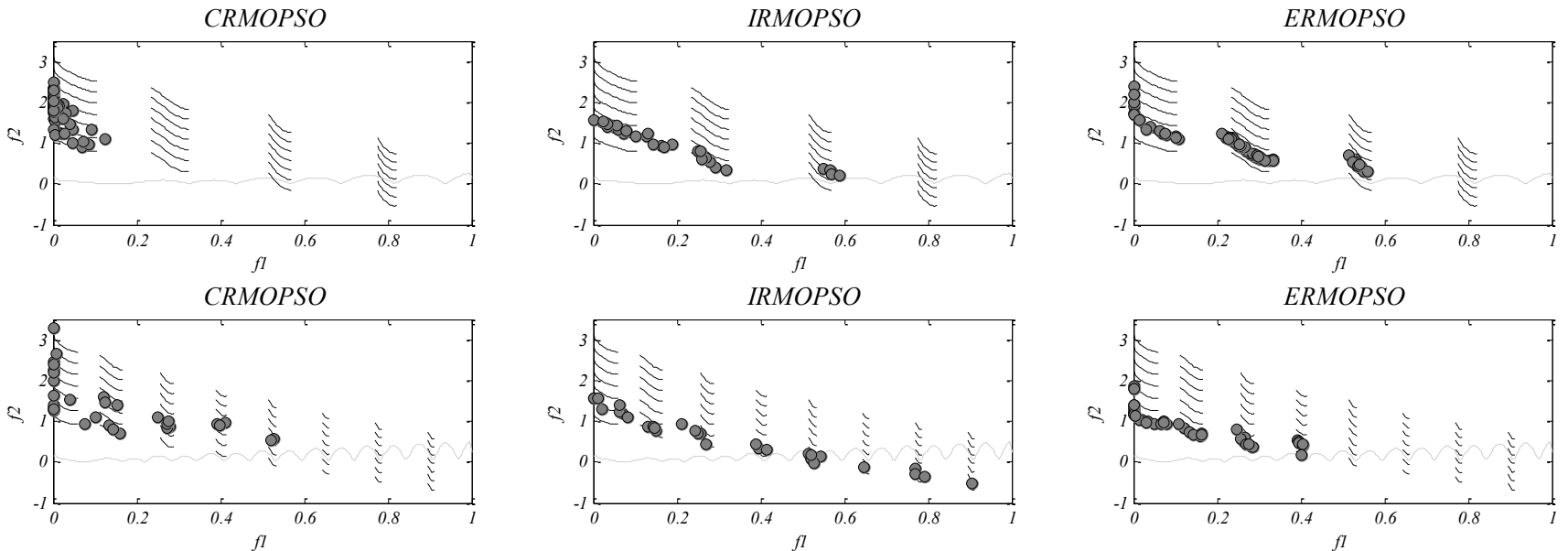

Figure 11: Robust fronts obtained for MTP9 and MTP10, one test case per row.

MOPSO algorithm. As discussed in the problem statement of this paper, there is a significant number of times during the optimisation that fewer neighbouring solutions mislead an algorithm toward non-robust regions. The archive-based mechanism of IRMOPSO deteriorated on these problems, with cases in which a non-robust solution entered the archive and was never dominated by robust solutions outside the archive. In contrast ERMOPSO which has an explicit averaging mechanism never favoured a non-robust solution. This caused a welldistributed robust front in Figure 10 for ERMOPSO on MTP6. The robust solutions obtained by CRMOPSO are very competitive with those found by ERMOPSO. TABLE 2 shows that CR- 
MOPSO had superior convergence, coverage, and success ratio on MTP6.

The approximate solutions of the algorithms on MTP7 were somewhat different; the coverage of solutions obtained by ERMOPSO was very low and the CRMOPSO algorithm also found non-robust solutions. Generally speaking, approximation of a concave front is more challenging, and this phenomenon can be seen for the MTP7 test function. The IRMOPSO algorithm again failed to estimate the robust Pareto optimal front, whereas CRMOPSO and ERMOPSO tended to find robust solutions.

MTP8 has two fronts with opposite shapes: a convex global front and a concave robust/local front. The results of algorithms on this test function show the difficulty of MTP8 as none of the algorithms approximate the entire robust front. In Figure 10, some resolution of the robust front can be observed in the solutions obtained by both CRMOPSO and ERMOPSO. The CRMOPSO algorithm shows a slightly greater tendency to correct selection in this case. Deb asserted that different shapes for front makes the search space very difficult for optimisation algorithms in multi-objective benchmark problems [70], and it is the reason for the poor performance of all algorithms on MTP8.

The last two test functions, MTP9 and MTP10, have multiple discontinuous local fronts. They are the most difficult test cases for the algorithms. Note that the robustness increases from right to left and bottom to top in the figure. The results of TABLE 2 and Figure 11 show that the proposed CRMOPSO algorithm was the best in terms of favouring robust regions of the fronts. The Pareto optimal solutions obtained by CRMOPSO for MTP9 indicate that there is more coverage on the most robust region of the local fronts. However, IRMOPSO and ERMOPSO do not display such behaviours. The results of algorithms on MTP10, which has more discontinuous robust regions, were also consistent with those of MTP9: the CRMOPSO algorithm was able to find more robust solutions.

The average and standard deviations show that the proposed algorithm is able to provide very promising overall results. To see if this superiority is statistically significant, Wilcoxon ranksum test is conducted considering 5\% significance level. For the statistical test, the best algorithm in each test function is chosen and compared with other algorithms independently. For example, if the best algorithm is CRMOPSO, a pairwise comparison is done between CRMOPSO/IRMOPSO and CRMOPSO/ERMOPSO. Note that since the best algorithm cannot be compared with itself, N/A has been written for the best algorithm in each function which stands for Not Applicable. The pvalues obtained are shown in TABLE $3 ; p \geq 0.5$ are underlined. The results of this table show that the proposed CRMOPSO algorithm significantly outperforms IRMOPSO on the majority of test cases for all performance measures. This algorithm is very competitive compared to the ERMOPSO algorithm as well. Since the Wilcoxon ranksum test considers each of 30 independent runs, these results prove that the proposed algorithm benefits from high reliability in finding robust Pareto optimal solutions without any extra function evaluation.

To further investigate the efficiency of CRMOPSO, we determined the number of times that a solution dominated an archive member but was not allowed to enter the archive with the proposed confidence measure, as shown in TABLE 4. This table shows that a significant number of times during optimisation (approximately 19\% of attempts) the proposed confidencebased Pareto optimality concepts prevented solutions with low confidence from being added to the archive. Firstly, these results demonstrate that often IRMOPSO was likely to make unreliable decisions and consequently favour non-robust solutions. Secondly, the proposed method is able to prevent unreliable decisions throughout optimisation, providing more reliability than the current archive-based robustness-handling methods.

In summary, the results show that the convergence of CRMOPSO is slower than IRMOPSO because less confident solutions were ignored over the course of iterations. Although this prevents CRMOPSO from providing superior results on uni-modal test functions, it can be very helpful in optimising real problems since CRMOPSO has less probability of premature convergence toward non-robust regions compared to IRMOPSO. The results of CRMOPSO were also superior to ERMOPSO on the majority of test functions. The proposed performance indicators quantitatively showed the greater coverage and success ratio of the CRMOPSO algorithm. In addition, the proposed algorithm showed very good convergence on the robust regions of challenging, multi-modal test cases.

\section{Real Application: Marine Propeller Design}

\subsection{Problem formulation}

There are two main objectives in propeller design: efficiency versus cavitation. Efficiency is the amount of motor power that is converted to thrust and should be maximised. Cavitation is the effects of local shock waves caused by the bubbles formed around a propeller that should be minimised. The shape of a blade contributes most in these two objectives and is the main focus of this work. In order to find the final geometrical shape of the blade, standard National Advisory Committee for Aeronautics (NACA) airfoils are chosen as illustrated in Fig. 12. This figure shows that thickness and chord length are the main parameters of each airfoil. Ten airfoils are selected along the blade, so there are 20 parameters to be optimised for this problem.

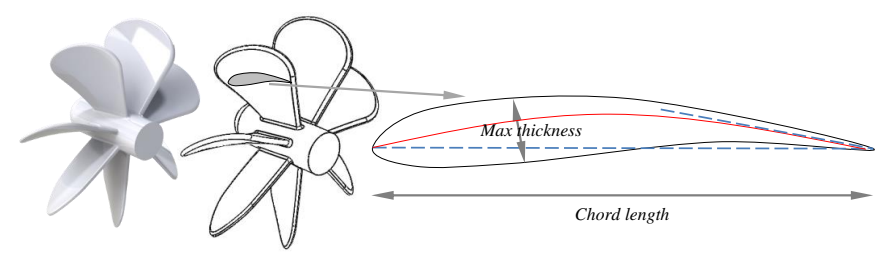

Figure 12: NACA a=0.8 meanline and NACA 65A010 thickness

The problem is formulated as follows:

$$
\text { Suppose }: \vec{X}=\left(T_{i}, C_{i}\right), i=1,2, \ldots, 10
$$

$$
\text { Maximise : } \eta(\vec{X})
$$


Table 3: P-values obtained after conducting Wilcoxon ranksum test on the results in Table 2. Note that p-values greater than or equal to 0.5 are underlined.

\begin{tabular}{cccccccccc}
\hline \multicolumn{3}{c}{ CRMOPSO } & \multicolumn{3}{c}{ IRMOPSO } & \multicolumn{3}{c}{ ERMOPSO } \\
\hline & $\Xi$ & $\Phi$ & $\Gamma$ & $\Xi$ & $\Phi$ & $\Gamma$ & $\Xi$ & $\Phi$ & $\Gamma$ \\
MTP1 & 0.0058 & 0.0003 & N/A & N/A & N/A & 0.0003 & 0.0022 & 0.0027 & 0.0002 \\
MTP2 & 0.0002 & 0.0079 & N/A & 0.0013 & 0.0002 & $\underline{0.1041}$ & N/A & N/A & 0.0211 \\
MTP3 & 0.0010 & $\underline{0.3629}$ & N/A & N/A & 0.0336 & 0.0010 & $\underline{0.3447}$ & N/A & $\underline{0.0638}$ \\
MTP4 & N/A & N/A & 0.0002 & 0.0439 & 0.0229 & $\underline{0.9097}$ & 0.0002 & $\underline{0.7909}$ & N/A \\
MTP5 & N/A & 0.0171 & $\underline{0.1859}$ & 0.0439 & N/A & N/A & 0.0036 & $\underline{0.5665}$ & $\underline{0.2730}$ \\
MTP6 & N/A & N/A & 0.0002 & 0.0002 & $\underline{0.1971}$ & N/A & 0.0002 & $\underline{0.0957}$ & 0.0138 \\
MTP7 & N/A & N/A & N/A & 0.0004 & $\underline{0.1197}$ & 0.0028 & 0.0257 & $\underline{0.8793}$ & 0.0002 \\
MTP8 & N/A & N/A & 0.0173 & $\underline{0.6776}$ & 0.0029 & N/A & 0.0046 & $\underline{0.1810}$ & 0.0439 \\
MTP9 & N/A & N/A & $\underline{0.6776}$ & $\underline{0.2730}$ & 0.0293 & $\underline{0.5437}$ & 0.0376 & $\underline{0.2852}$ & N/A \\
MTP10 & N/A & $\underline{0.5644}$ & $\underline{0.4495}$ & 0.0002 & 0.0008 & 0.0171 & 0.0002 & N/A & N/A \\
\hline
\end{tabular}

Table 4: Number of times that the proposed confidence-based Pareto dominance prevented a solution entering the archive

\begin{tabular}{c|c}
\hline Test function & $\left(P_{i} \prec\right.$ Archive $\left._{m}\right) \wedge\left(P_{i} \nprec_{c}\right.$ Archive $\left._{m}\right)$ \\
\hline MTP1 & $18,918 / 100,000$ \\
\hline MTP2 & $28,210 / 100,000$ \\
\hline MTP3 & $19,240 / 100,000$ \\
\hline MTP4 & $17,157 / 100,000$ \\
\hline MTP5 & $13,938 / 100,000$ \\
\hline MTP6 & $14,703 / 100,000$ \\
\hline MTP7 & $15,768 / 100,000$ \\
\hline MTP8 & $17,054 / 100,000$ \\
\hline MTP9 & $26,566 / 100,000$ \\
\hline MTP10 & $17,570 / 100,000$ \\
\hline
\end{tabular}

$$
\text { Minimise : } V_{c}(\vec{X})
$$

$$
\text { Subject to : Thrust } \geq 40000
$$

where $T_{i}$ and $C_{i}$ shows the thickness and chord length of the $i$-th airfoil and $V_{c}$ is a function to calculate the cavitation.

\subsection{Uncertainties in the structural parameters}

The most common uncertainties in propeller design may occur in the structural parameters and operating condition. The former type of uncertainty mostly originates from manufacturing errors. This work considers the maximum permitted errors ( $\delta=1.5 \%$ ), according to ISO 484/2-1981, that can alter the optimal values obtained by MOPSO and CRMOPSO. .

The MOPSO algorithm is first employed to estimate the true Pareto optimal front for this problem. The solutions obtained will then be perturbed considering $\delta=1.5 \%$ manufacturing error. The results are given in Fig. 13. Note that we re-calculate the objectives for each Pareto optimal solutions perturbed.

It is evident in this figure that uncertainties in the thickness and chord length of airfoils substantially degrade the expected efficiency. Reduction in efficiency translates directly to increased fuel consumption, the biggest cost in marine shipping. Also, it seems that the changes in cavitation is more than efficiency, which remarkably reduces the lifespan of a propeller. There results strongly show that perturbations in structural parameters of a propeller are able to negatively impacts the expected outputs. Therefore, it is essential to consider them as undesirable inputs to look for robust solutions to minimise their effects.

\subsection{Confidence-based Robust optimisation of marine pro- pellers}

The preceding subsection showed the significant negative impacts of perturbations in parameters on both objectives of the propeller design problem. The proposed confident-based robust multi-objective perspective in this work has been designed to handle such perturbations without the need for extra function evaluation. Therefore, this subsection employs the proposed CRMOPSO algorithm for finding robust Pareto optimal solutions for the propeller design problem.

The experimental set up is identical to that of the preceding section, in which 100 particles are utilised and allowed to search for the robust Pareto optimal solutions over 200 number of iterations. The maximum level of perturbation is also considered as $1.5 \%$ as per ISO $484 / 2-1981$. The CRMOPSO algorithm was run 5 times and the best approximated robust front is illustrated in Fig. 14. Note that the best Pareto optimal front obtained by MOPSO is illustrated in this figure as well for comparison.

It may be seen in this figure that the robust Pareto optimal front is completely dominated by the global Pareto optimal front. The coverage of the robust front is also lower than the global front. In order to see the significant of the results, the amount of excessive fuel consumptions that can be raised in case of $1.5 \%$ perturbation during manufacturing are calculated for the solutions obtained by both algorithms and illustrated in Fig. 15.

This figure shows that the discrepancy of fuel consumption is much lower for the designs obtained by CRMOPSO algorithm. The average, minimum, and maximum of the excessive fuel consumption is almost half for the solutions obtained by the CRMOPSO algorithm. Due to the difficulty of the propeller design problem and importance of uncertainties, these results strongly evidence the merits of the proposed confidence-based robust optimisation. It is worth highlighting here that the robust Pareto optimal solutions are obtained without even a single extra function evaluation.

To further investigate the effectiveness of the proposed CRMOPSO, this algorithm is employed to find the robust optimal 

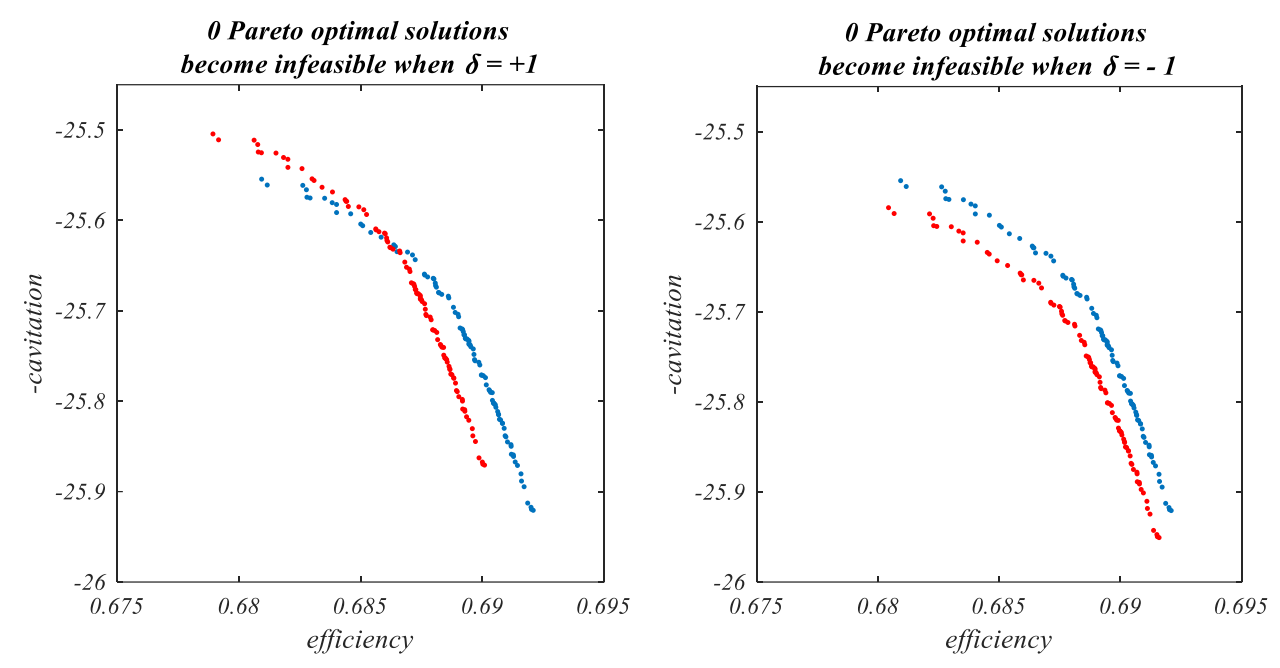

Figure 13: Pareto optimal fronts when (left) $\delta=+1.5 \%$ (right) $\delta=-1.5 \%$ perturbations in parameters. Original Pareto optimal front is shown in blue, perturbed results in red.

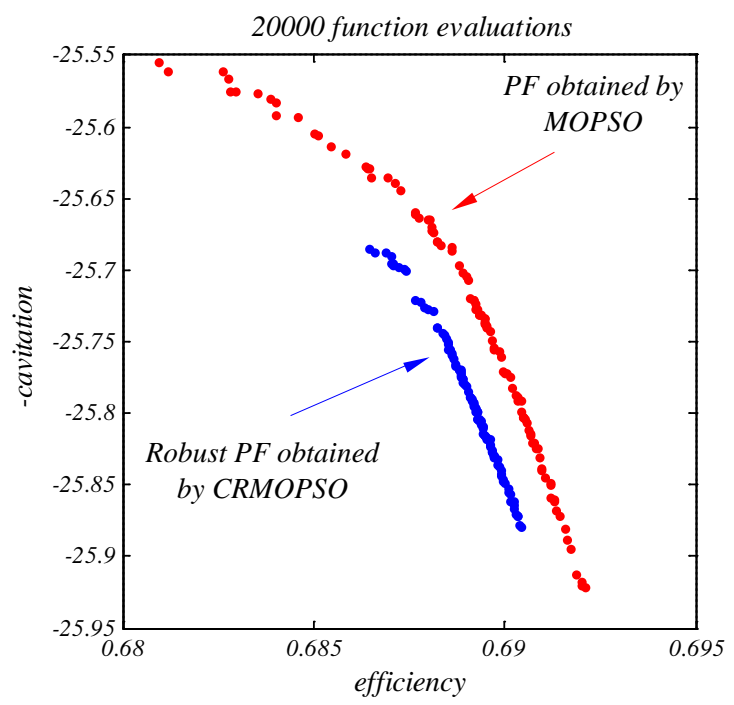

Figure 14: Robust front obtained by CRMOPSO versus global front obtained by MOPSO

values for Revolution Per Minute (RPM) as the main operating condition. MOPSO is first required to approximate the global Pareto optimal front for this problem with parameterising the RPM. An equal number of particles and iterations are then employed to determine the robust front and robust optimal values for RPM using CRMOPSO. The results are illustrated in Fig. 16.

As may be seen in Fig. 16 the major part of the robust Pareto front is dominated by the global front. The distributions of both fronts are almost identical. A small portion of robust front overlaps with global front. Therefore, the robust front is of type C. To observe the range of RPMs in both fronts, Fig. 17 is provided.

This figure shows that the range of RPM obtained by both algorithms is evidently different. It may be seen that the range of RPM tends to be higher in the Pareto optimal solutions ob- tained by CRMOPSO. Another interesting pattern is that half of the Pareto optimal solutions obtained by MOSPO have RPM of 170. However, the results of CRMOPO in Fig. 17 show that 170 is not a robust RPM since a few of the solutions have this value for their RPMs. The range of RPMs in CRMOPSO is wider than that of MOPSO. This is due to the intrisic higher exploration of the CRMOPSO. Since less non-dominated solutions are alloweded to enter the archive, the exploitation is less and exploration is higher than the normal MOPO, which results in finding a wider range of RPMs.

To observe the impacts of the perturbation on the Pareto optimal solutions obtained by both of the algorithms, Fig. 18 is provided. Note that the RPM is only perturbed in this experiment and other parameters are fixed. This figure shows that the average, maximum, and minimum fuel consumption discrepancy of the robust Pareto optimal solutions obtained by CRMOPSO algorithm is better that those of MOPSO. These results again strongly prove the merits of the proposed confidence-based robust optimisation approach in finding robust solutions that are less sensitive to perturbations in parameters.

In summary, the results of this section highly demonstrate that effectiveness of the proposed robust optimisation approach in finding robust optimal solutions for computationally expensive real-world problems with unknown search spaces. This is due to the proposed confidence-based Pareto optimal dominance that allows robust algorithms to reliably compare solutions and decide about their robustness during optimisation without the need for even one extra function evaluation.

\section{Conclusion}

This paper proposed a novel approach for searching for robust solutions. A confidence metric was first employed to measure the confidence of search agents based on the status of previously sampled neighbouring solutions in the parameter space. Confidence-based Pareto optimality concepts were 

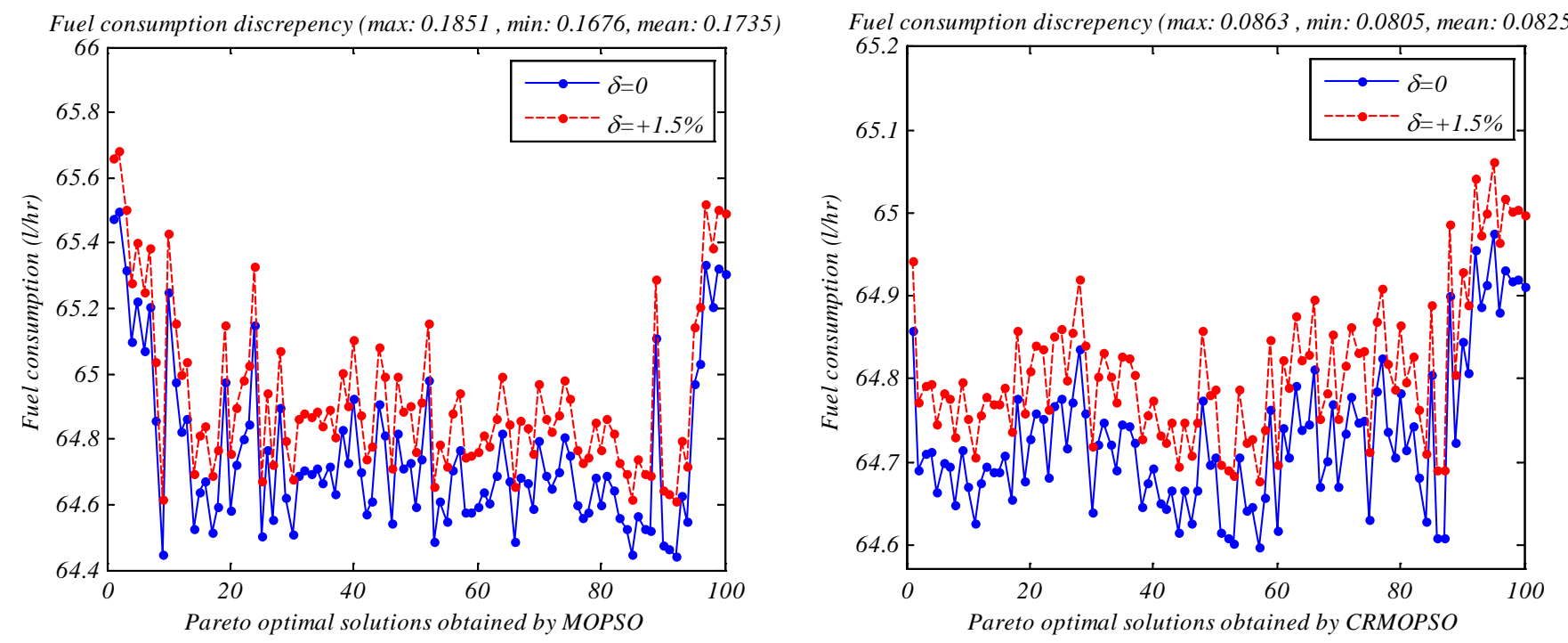

Figure 15: Fuel consumption discrepancy in case of perturbation in all of the structural parameters for both PF obtained by MOPSO and RPF obtained by CRMOPSO

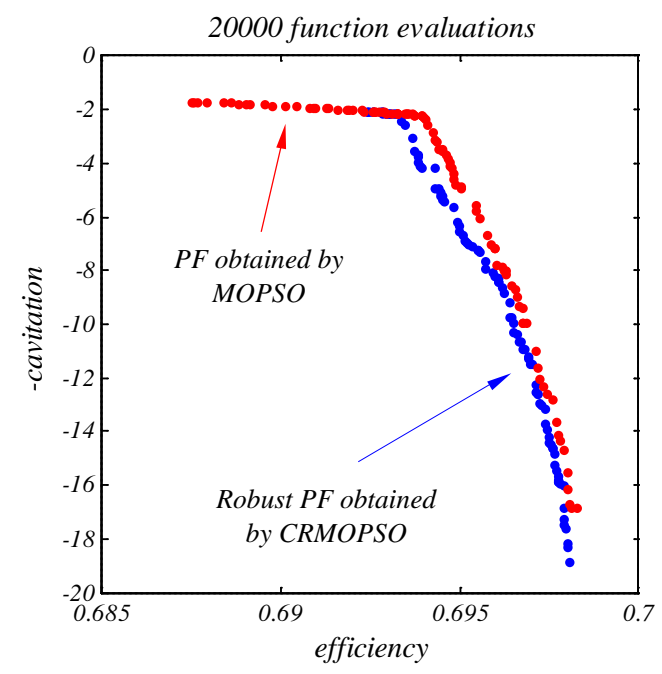

Figure 16: Global and robust Pareto optimal fronts obtained by MOPSO and CRMOPSO when RPM is also a variable

then proposed using the confidence metric. The confidencebased Pareto dominance was integrated with the archive update mechanism of RMOPSO as a case study. The paper also presented three performance indicators to quantitatively compare the performance of the proposed method. Due to the simplicity of the current robust multi-objective test functions, seven new, challenging test functions were generated within two general frameworks for the purpose of this study. The results showed that the novel approach proposed is able to deliver very promising results in terms of convergence, coverage, and proportion of robust Pareto optimal solutions obtained. The applicability of the proposed approach was confirmed by approximating robust Pareto optimal front for a marine propeller design problem as well.

The findings demonstrated the value of the proposed con-

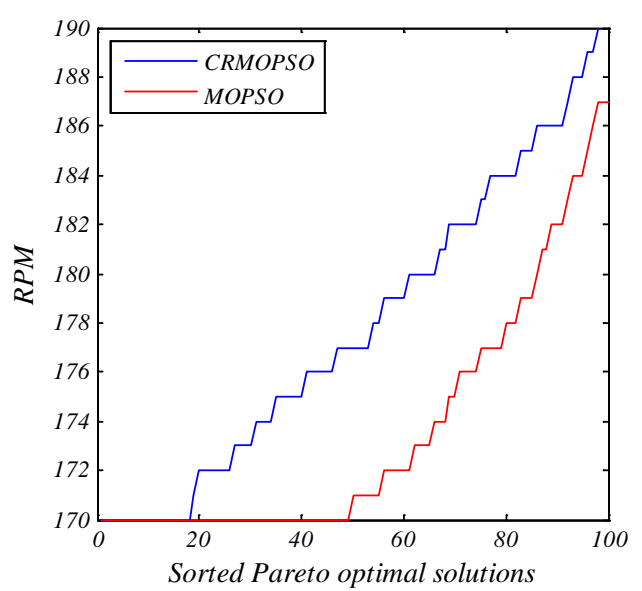

Figure 17: Optimal and robust optimal values for RPM (note that there are 98 robust Pareto optimal solutions and 100 global optimal solutions)

cepts:

- The proposed $C$ measure is able to effectively calculate the confidence level of search agents derives from the status of previously sampled neighbouring solutions.

- The proposed confidence-based Pareto optimality provides the opportunity to design different CRMO mechanisms for meta-heuristics.

- The proposed confidence-based Pareto optimality improves the reliability of archive-based robust optimisation techniques.

- CRMO is able to confidently find robust solutions.

- CRMO is computationally cheap since there is no need for additional true function evaluations. 

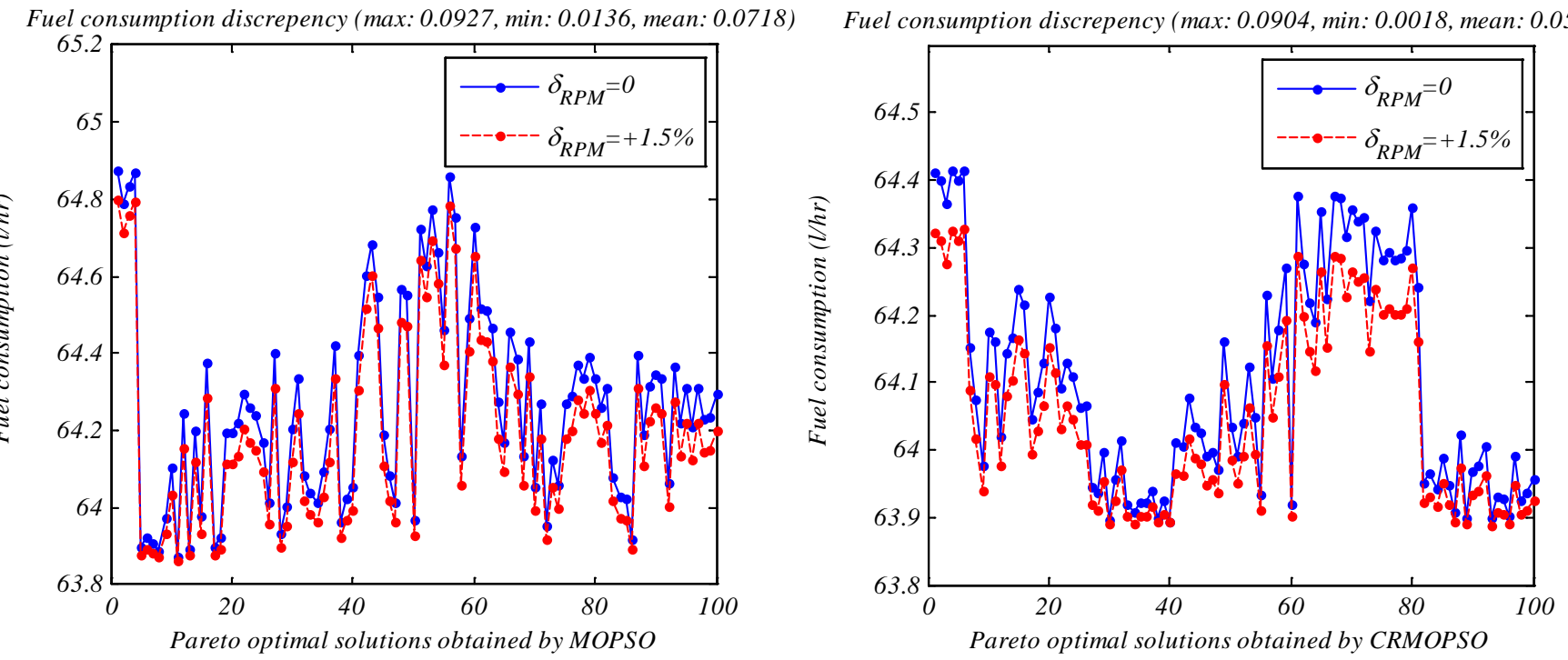

Figure 18: Fuel consumption discrepancy in case of perturbation in RPM for both PF obtained by MOPSO and RPF obtained by CRMOPSO

- CRMO is able to approximate different types of true robust Pareto optimal fronts.

- CRMO is suitable for computationally expensive realworld problems.

For future work, it would be interesting to investigate the merits of CRMO in constrained robust multi- and manyobjective optimisation. The authors also intend to apply the algorithms developed to real-world problems in engineering design.

\section{References}

[1] K. Deb and H. Gupta. Introducing robustness in multi-objective optimization. Evolutionary Computation, 14(4):463-494, 2006.

[2] C. K. Goh, K. C. Tan, C. Y. Cheong, and Y. S. Ong. An investigation on noise-induced features in robust evolutionary multi-objective optimization. Expert Systems with Applications, 37(8):5960-5980, 2010.

[3] C. K. Goh and K. C. Tan. Robust evolutionary multi-objective optimization. In Evolutionary Multi-objective Optimization in Uncertain Environments, pages 189-211. Springer, 2009.

[4] Y. Jin and J. Branke. Evolutionary optimization in uncertain environments-a survey. IEEE Transactions on Evolutionary Computation, 9(3):303-317, 2005.

[5] J. Ferreira, C. M. Fonseca, J. A. Covas, and A. Gaspar-Cunha. Evolutionary multi-objective robust optimization. Advances in evolutionary algorithms, pages 261-278, 2008.

[6] A. Gaspar-Cunha, J. Ferreira, and G. Recio. Evolutionary robustness analysis for multi-objective optimization: benchmark problems. Structural and Multidisciplinary Optimization, pages 1-23, 2013.

[7] Y. Jin, M. Olhofer, and B. Sendhoff. A framework for evolutionary optimization with approximate fitness functions. IEEE Transactions on Evolutionary Computation, 6(5):481-494, 2002.

[8] C. A. Coello Coello, G. T. Pulido, and M. S. Lechuga. Handling multiple objectives with particle swarm optimization. IEEE Transactions on Evolutionary Computation, 8(3):256-279, 2004.

[9] D. Chen, F. Zou, and J. Wang. A multi-objective endocrine pso algorithm and application. Applied Soft Computing, 11(8):4508-4520.

[10] G. Chiandussi, M. Codegone, S. Ferrero, and F. E. Varesio. Comparison of multi-objective optimization methodologies for engineering applications. Computers and Mathematics with Applications, 63(5):912-942.
[11] K. Deb, A. Pratap, S. Agarwal, and T. Meyarivan. A fast and elitist multiobjective genetic algorithm: Nsga-ii. IEEE Transactions on Evolutionary Computation, 6(2):182-197, 2002.

[12] I. Y. Kim and O. L. De Weck. Adaptive weighted-sum method for biobjective optimization: Pareto front generation. Structural and Multidisciplinary Optimization, 29(2):149-158, 2005.

[13] A. Messac and C. A. Mattson. Generating well-distributed sets of pareto points for engineering design using physical programming. Optimization and Engineering, 3(4):431-450, 2002.

[14] I. Das and J. E. Dennis. Normal-boundary intersection: A new method for generating the pareto surface in nonlinear multicriteria optimization problems. SIAM Journal on Optimization, 8(3):631-657, 1998.

[15] K. Deb. Advances in evolutionary multi-objective optimization. Search Based Software Engineering, pages 1-26, 2012.

[16] N. Srinivas and K. Deb. Muiltiobjective optimization using nondominated sorting in genetic algorithms. Evolutionary computation, 2(3):221-248, 1994.

[17] H. G. Beyer and B. Sendhoff. Robust optimization-a comprehensive survey. Computer methods in applied mechanics and engineering, 196(3334):3190-3218, 2007.

[18] K. Deb and H. Gupta. Searching for robust pareto-optimal solutions in multi-objective optimization. Lecture Notes in Computer Science, 3410:150-164, 2005.

[19] A. Gaspar-Cunha and J. Covas. Robustness in multi-objective optimization using evolutionary algorithms. Computational Optimization and Applications, 39(1):75-96, 2008.

[20] C. Barrico and C.H. Antunes. Robustness analysis in multi-objective optimization using a degree of robustness concept. pages 1887-1892. IEEE, 1892.

[21] C. Barrico and C.H. Antunes. A new approach to robustness analysis in multi-objective optimization. In 7th International Conference on MultiObjective Programming and Goal Programming, pages 12-14, 2006.

[22] D. Wiesmann, U. Hammel, and T. Back. Robust design of multilayer optical coatings by means of evolutionary algorithms. IEEE Transactions on Evolutionary Computation, 2(4):162-167, 1998.

[23] Y. Jin and B. Sendhoff. Trade-off between performance and robustness: An evolutionary multiobjective approach. pages 68-68. Springer, 2003.

[24] S. Tsutsui and A. Ghosh. Genetic algorithms with a robust solution searching scheme. IEEE Transactions on Evolutionary Computation, 1(3):201-208, 1997.

[25] T. Ray. Constrained robust optimal design using a multiobjective evolutionary algorithm. In 2002 Congress on Evolutionary Computation, volume 1, pages 419-424, 2002.

[26] F. López-Monzalvo and C. A. Brizuela. An experimental analysis of 
the p-median problem under uncertainty: an evolutionary algorithm approach. European Journal of Industrial Engineering, 8(4):554-578, 2014.

[27] D. Brockhoff, T. Friedrich, N. Hebbinghaus, C. Klein, F. Neumann, and E. Zitzler. On the effects of adding objectives to plateau functions. IEEE Transactions on Evolutionary Computation, 13(3):591-603, 2009.

[28] D. Brockhoff, T. Friedrich, N. Hebbinghaus, Christian Klein, F. Neumann, and E. Zitzler. Do additional objectives make a problem harder? In 2007 conference on Genetic and evolutionary computation, pages 765772. ACM, 2007.

[29] J. Esteban Diaz and J. Handl. Implicit and explicit averaging strategies for simulation-based optimization of a real-world production planning problem. Informatica, 39(2):161-168, 2015.

[30] J. E. Fieldsend. Elite Accumulative Sampling Strategies for Noisy Multiobjective Optimisation, pages 172-186. Springer, 2015.

[31] J. E. Fieldsend and R. M. Everson. The rolling tide evolutionary algorithm: A multiobjective optimizer for noisy optimization problems. IEEE Transactions on Evolutionary Computation, 19(1):103-117, 2015.

[32] K. Alyahya, K. Doherty, J. E. Fieldsend, and O. E. Akman. On the exploitation of search history and accumulative sampling in robust optimisation. In 2017 Conference Genetic and Evolutionary Computation, pages 185-186, 2017.

[33] J. Branke and X. Fei. Efficient sampling when searching for robust solutions. In International Conference on Parallel Problem Solving from Nature, pages 237-246. Springer, 2016.

[34] I. R. Meneghini, F. G. Guimarães, and A. Gaspar-Cunha. Competitive coevolutionary algorithm for robust multi-objective optimization: The worst case minimization. In 2016 IEEE Congress on Evolutionary Computation, pages 586-593. IEEE, 2016.

[35] C. L. Sabioni, M. F. de Oliveira Ribeiro, and J. A. de Vasconcelos. Decision maker iterative-based framework for multiobjective robust optimization. Neurocomputing, 242:113-130, 2017.

[36] A. Cervantes, D. Quintana, and G. Recio. Efficient dynamic resampling for dominance-based multiobjective evolutionary optimization. Engineering Optimization, 49(2):311-327, 2017.

[37] W. Zhang, Y. Wang, C. Wang, L. Xiao, and M. Gen. Fast multiobjective hybrid evolutionary algorithm based on mixed sampling strategy. In International Conference on Management Science and Engineering Management, pages 106-116. Springer, 2017.

[38] Q. Zhou, P. Jiang, X. Huang, F. Zhang, and T. Zhou. A multi-objective robust optimization approach based on gaussian process model. Structural and Multidisciplinary Optimization, pages 1-21, 2017.

[39] Q. Zhou, P. Zhou, H. Shao, H. Zhou, and J. Hu. An on-line kriging metamodel assisted robust optimization approach under interval uncertainty. Engineering Computations, 34(2):420-446, 2017.

[40] L. Cao, P. Jiang, Z. Chen, Q. Zhou, and H. Zhou. Metamodel assisted robust optimization under interval uncertainly based on reverse model. IFAC-PapersOnLine, 48(28):1178-1183, 2015

[41] Q. Zhou, X. Shao, P. Jiang, H. Zhou, L. Cao, and L. Zhang. A deterministic robust optimisation method under interval uncertainty based on the reverse model. Journal of Engineering Design, 26(10-12):416-444, 2015

[42] N. Hu, B. Duan, H. Cao, and Y. Zong. Robust optimization with convex model considering bounded constraints on performance variation. Structural and Multidisciplinary Optimization, pages 1-11, 2017.

[43] M. Kaedi and C. W. Ahn. Robust optimization using bayesian optimization algorithm: Early detection of non-robust solutions. Applied Soft Computing, 2017.

[44] Yaochu Jin, Ke Tang, Xin Yu, Bernhard Sendhoff, and Xin Yao. A framework for finding robust optimal solutions over time. Memetic Computing, 5(1):3-18, 2013.

[45] Yuanjun Huang, Yongsheng Ding, Kuangrong Hao, and Yaochu Jin. A multi-objective approach to robust optimization over time considering switching cost. Information Sciences, 394:183-197, 2017.

[46] Guangyong Sun, Guangyao Li, Shiwei Zhou, Hongzhou Li, Shujuan Hou, and Qing Li. Crashworthiness design of vehicle by using multiobjective robust optimization. Structural and Multidisciplinary Optimization, 44(1):99-110, 2011.

[47] Xianguang Gu, Guangyong Sun, Guangyao Li, Lichen Mao, and Qing Li. A comparative study on multiobjective reliable and robust optimization for crashworthiness design of vehicle structure. Structural and Multidis- ciplinary Optimization, 48(3):669-684, 2013

[48] Guangyong Sun, Xueguan Song, Seokheum Baek, and Qing Li. Robust optimization of foam-filled thin-walled structure based on sequential kriging metamodel. Structural and Multidisciplinary Optimization, 49(6):897-913, 2014

[49] Guangyong Sun, Huile Zhang, Ruoyu Wang, Xiaojiang Lv, and Qing Li. Multiobjective reliability-based optimization for crashworthy structures coupled with metal forming process. Structural and Multidisciplinary Optimization, 56(6):1571-1587, 2017.

[50] Guangyong Sun, Huile Zhang, Jianguang Fang, Guangyao Li, and Qing Li. Multi-objective and multi-case reliability-based design optimization for tailor rolled blank (trb) structures. Structural and Multidisciplinary Optimization, 55(5):1899-1916, 2017.

[51] Xueguan Song, Guangyong Sun, and Qing Li. Sensitivity analysis and reliability based design optimization for high-strength steel tailor welded thin-walled structures under crashworthiness. Thin-Walled Structures, 109:132-142, 2016

[52] Guangyong Sun, Guangyao Li, Shiwei Zhou, Wei Xu, Xujing Yang, and Qing Li. Multi-fidelity optimization for sheet metal forming process. Structural and Multidisciplinary Optimization, 44(1):111-124, 2011.

[53] Guangyong Sun, Huile Zhang, Jianguang Fang, Guangyao Li, and Qing Li. A new multi-objective discrete robust optimization algorithm for engineering design. Applied Mathematical Modelling, 53:602-621, 2018.

[54] Wei Du, Yang Tang, Sunney Yung Sun Leung, Le Tong, Athanasios V Vasilakos, and Feng Qian. Robust order scheduling in the discrete manufacturing industry: A multiobjective optimization approach. IEEE Transactions on Industrial Informatics, 14(1):253-264, 2018.

[55] A. Saha, T. Ray, and W. Smith. Towards practical evolutionary robust multi-objective optimization. In 2011 IEEE Congress on Evolutionary Computation, pages 2123-2130. IEEE, 2011.

[56] J. Branke. Creating robust solutions by means of evolutionary algorithms. In Parallel Problem Solving from NaturePPSN V, pages 119-128. Springer, 1998.

[57] Y. S. Ong, P. B. Nair, and K. Y. Lum. Max-min surrogate-assisted evolutionary algorithm for robust design. IEEE Transactions on Evolutionary Computation, 10(4):392-404, 2006.

[58] T. Ray and W. Smith. A surrogate assisted parallel multiobjective evolutionary algorithm for robust engineering design. Engineering Optimization, 38(8):997-1011, 2006.

[59] Y. Jin. Surrogate-assisted evolutionary computation: Recent advances and future challenges. Swarm and Evolutionary Computation, 1(2):6170, 2011.

[60] C. E. J. Dippel. Using particle swarm optimization for finding robust optima. Leiden University, 2008.

[61] S. Mirjalili, A. Lewis, and S. Mostaghim. Confidence measure: A novel metric for robust meta-heuristic optimisation algorithms. Information Sciences, 317:114-142, 2015.

[62] S. Mirjalili and A. Lewis. Novel frameworks for creating robust multiobjective benchmark problems. Information Sciences, 300:158-192, 2015 .

[63] S. Mirjalili and A. Lewis. Hindrances for robust multi-objective test problems. Applied Soft Computing, 35:333-348, 2015.

[64] S. Mirjalili. Shifted robust multi-objective test problems. Structural and Multidisciplinary Optimization, 52(1):217-226, 2015.

[65] S. Mirjalili and A. Lewis. Novel performance metrics for robust multiobjective optimization algorithms. Swarm and Evolutionary Computation, 21:1-23, 2015.

[66] T. Erfani and S. Utyuzhnikov. Control of robust design in multiobjective optimization under uncertainties. Structural and Multidisciplinary Optimization, 45(2):247-256, 2012.

[67] A. Gaspar-Cunha and J. A. Covas. Robustness using Multi-Objective Evolutionary Algorithms, pages 353-362. Springer, 2006.

[68] E. Zitzler, L. Thiele, M. Laumanns, C. M Fonseca, and V. G. Da Fonseca. Performance assessment of multiobjective optimizers: An analysis and review. IEEE Transactions on Evolutionary Computation, 7(2):117-132, 2003.

[69] A. Lewis, S. Mostaghim, and I. Scriven. Asynchronous multiobjective optimisation in unreliable distributed environments, pages 5178. Springer, 2009.

[70] K. Deb. Multi-objective genetic algorithms: Problem difficulties and construction of test problems. Evolutionary computation, 7(3):205-230, 
